\title{
McSCIA: application of the Equivalence Theorem in a Monte Carlo radiative transfer model for spherical shell atmospheres
}

\author{
F. Spada ${ }^{1}$, M. C. Krol ${ }^{1,2,3}$, and P. Stammes ${ }^{4}$ \\ ${ }^{1}$ Institute for Marine and Atmospheric research Utrecht (IMAU), Utrecht, The Netherlands \\ ${ }^{2}$ Netherlands Institute for Space Research (SRON), Utrecht, The Netherlands \\ ${ }^{3}$ Meteorology and Air Quality, Wageningen University, The Netherlands \\ ${ }^{4}$ Koninklijk Nederlands Meteorologisch Instituut (KNMI), De Bilt, The Netherlands
}

Received: 10 November 2005 - Published in Atmos. Chem. Phys. Discuss.: 15 February 2006

Revised: 26 September 2006 - Accepted: 3 October 2006 - Published: 25 October 2006

\begin{abstract}
A new multiple-scattering Monte Carlo 3-D radiative transfer model named McSCIA (Monte Carlo for SCIAmachy) is presented. The backward technique is used to efficiently simulate narrow field of view instruments. The McSCIA algorithm has been formulated as a function of the Earth's radius, and can thus perform simulations for both plane-parallel and spherical atmospheres. The latter geometry is essential for the interpretation of limb satellite measurements, as performed by SCIAMACHY on board of ESA's Envisat. The model can simulate UV-vis-NIR radiation.
\end{abstract}

First the ray-tracing algorithm is presented in detail, and then successfully validated against literature references, both in plane-parallel and in spherical geometry. A simple 1-D model is used to explain two different ways of treating absorption. One method uses the single scattering albedo while the other uses the equivalence theorem. The equivalence theorem is based on a separation of absorption and scattering. It is shown that both methods give, in a statistical way, identical results for a wide variety of scenarios. Both absorption methods are included in McSCIA, and it is shown that also for a 3-D case both formulations give identical results. McSCIA limb profiles for atmospheres with and without absorption compare well with the one of the state of the art Monte Carlo radiative transfer model $\mathrm{MCC}++$.

A simplification of the photon statistics may lead to very fast calculations of absorption features in the atmosphere. However, these simplifications potentially introduce biases in the results. McSCIA does not use simplifications and is therefore a relatively slow implementation of the equivalence theorem.

Correspondence to: F. Spada

(francesco.spada@gmail.com)

\section{Introduction}

In recent years the chemical composition of the atmosphere has become an important concern (e.g. Jacob, 1999). Due to human activities the composition of the atmosphere is changing, not only on a local scale, but also on a global scale. Many of these changes are related to trace gases present in the atmosphere (e.g. $\mathrm{O}_{3}, \mathrm{NO}_{2}, \mathrm{CO}_{2}$ ). To increase our knowledge on sources and sinks of these trace gases long time series of measurements are needed on a global scale, which can not be provided by ground stations only. Therefore, there has recently been an increase in new satellites with new instruments and capabilities. The aim of these new instruments is to monitor our changing atmosphere and understand, in synergy with chemical models and ground observations, the mechanisms behind the changes in atmospheric composition.

Many satellite instruments are designed to sample the atmospheric composition using the UV-vis-NIR part of the spectrum. Employing the gas absorption spectral features it is possible to retrieve the total column gas concentration of $\mathrm{O}_{3}, \mathrm{NO}_{2}, \mathrm{SO}_{2}, \mathrm{CO}, \mathrm{CH}_{4}, \mathrm{CO}_{2}$ from the backscattered solar radiation. Using the backscattered UV-vis-NIR solar radiation, for instance, GOME on board of ESA's ERS-2 (Burrows et al., 1999) employs a differential optical absorption spectroscopy (DOAS) method to obtain global distributions of various trace gases. Also the more recent instruments SCIAMACHY (Bovensmann et al., 1999) (launched 1 March 2002 onboard of ESA's Envisat), and OMI (Levelt et al., 2006; Stammes et al., 1999) (launched 15 July 2004 on board of NASA's EOS-Aura) use DOAS-like techniques to retrieve trace gas concentrations.

Whereas GOME and OMI are nadir viewing instruments, SCIAMACHY scans the atmosphere also in limb mode, giving the possibility to obtain stratospheric profiles and consequently tropospheric trace gas columns.

Published by Copernicus GmbH on behalf of the European Geosciences Union. 
The limb view enables the retrieval of vertical profiles of trace gases (see e.g. Rusch et al., 1984; Mount et al., 1984; Flittner et al., 2000; Kaiser, 2002; von Savigny et al., 2003; Kaiser et al., 2004; Sioris et al., 2004; Rozanov et al., 2005; Segers et al., 2005), but it raises some radiative transfer (RT) modelling issues. While for nadir geometry a plane-parallel description of the atmosphere normally suffices, in limb it is essential to consider the sphericity of the Earth. One approach to solve this problem is the use of a Monte Carlo (MC) RT model (Oikarinen et al., 1999). Monte Carlo methods are in principle an accurate way of solving RT problems, and are often used as a benchmark for approximate approaches (e.g. Walter and Landgraf, 2005). All other approaches work with some assumption to solve the RT equation in spherical geometry (see Lenoble, 1985; Marshak and Davis, 2005, for a review of different methods). Despite the fact that a Monte Carlo approach can be very time consuming, it also gives statistical information that allows to evaluate the error of the results. Another advantage that will be explored in this paper is the possibility to separate scattering from absorption. The separate treatment of these processes is achieved using the Equivalence Theorem (ET). Although it was already introduced by Irvine (1964) and illustrated by van de Hulst (1980), only recently a rigourous implementation, also for nontrivial cases, was published (Partain et al., 2000). In short, the ET states that it does not matter whether the constituents doing the scattering and doing the absorption are identical, i.e. absorption can be treated as only happening at the scattering points or only along the photon paths. In previous works on ET (van de Hulst, 1980; Feigelson, 1984; Cahalan et al., 1994; Partain et al., 2000) normalised probability distribution functions (PDFs) of photons paths were derived, using Monte Carlo models. These PDFs were used to calculate the absorption in the atmosphere. In the studies of the radiation in a cloudy atmosphere presented by Feigelson (1984), she proposes instead to derive equivalent trajectories that can be convoluted with absorption profiles of gases. Since this approach is only valid in the weak absorption limit, this kind of treatment of absorption introduces a new approximation that we would like to avoid.

The purpose of this work is to introduce the MC RT model named McSCIA and to illustrate the use of the ET. For the first time we will show that the ET and the single scattering approach (SSA) give identical results in a 3-D spherical RT model, both in nadir and limb geometry.

We will describe in Sect. 2 the ray-tracing part of McSCIA, without absorption, and perform a validation (Sect. 3) of this module. We will introduce absorption in Sect. 4 and illustrate the two approaches that we will exploit in the paper: the SSA and the ET. After a theoretical illustration on how to apply absorption in a MC model (Sect. 4.1), we will use a simple 1-D model, in Sect. 4.2, to introduce the reader to a MC RT model with absorption. We will show that the SSA and the ET approach give identical results in 1-D. The validation of the 3-D implementation of McSCIA against the state-of-the-art MC RT model MCC++ (Postylyakov, 2004) will be done in Sect. 5, where we will show that the SSA and ET approach give statistically the same results in a wide variety of scenarios. After a discussion in Sect. 7 on the earlier use of the ET and the comparison with our approach, we will summarise in Sect. 8 our results and draw some conclusions.

\section{McSCIA ray-tracing module}

McSCIA is a scalar (no polarisation) backward (or timereversed, or adjoint) MC RT model (see Fig. 1), in which photons are tracked from their starting position in the satellite, until the end their trajectories in space. The implementation is similar to SIRO (Oikarinen et al., 1999), but the refractive bending is not implemented. The basis of MC (see also Cashwell and Everett, 1959; Spanier and Gelbard, 1969; Lenoble, 1985; Marshak and Davis, 2005) is that every interaction of a photon in the atmosphere can be described via a probability density function (PDF), the integral of which can be linked to a random number $\mathcal{R}$ (see the Appendix for some examples). By generating enough random realisations, the physical process can be simulated in a statistical sense. Once the probability of one event is known, it is possible to calculate the probability of a sequence of events as a Markov chain, because the events are independent.

For a more in depth description of the ray-tracing module, please refer to Fig. 2 and Appendix A4.

The whole ray-tracing algorithm has been formulated as a function of the Earth's radius $R_{\text {earth }}$. This enables McSCIA to increase the Earth's radius to very large values, effectively resulting in a plane-parallel atmospheric model. In this way a validation of the backward MC algorithm with plane-parallel RTMs is a straightforward exercise.

\section{Scattering in a spherical atmosphere: validation of ray-tracing module}

The current implementation of the model atmosphere consists of an arbitrary number of homogeneous spherical shells. The depth of each layer is specified independently. The model can treat molecular scattering (Rayleigh phase function, see Eq. A9) and aerosol and droplet scattering (Henyey-Greenstein phase function with asymmetry parameter $0 \leq g \leq 1$, see Eq. A13). Since absorption is treated later (see Sect. 4), here it is assumed that the atmosphere is conservatively scattering, so the single scattering albedo (SSA), $\omega$, is set to one. The ground reflection is assumed Lambertian, and the ground albedo can have a 2-D variability (see Sect. A3.3). Since absorption will be considered in Sect. 4, surface reflection is assumed, just for now, to be conservative. The changes in the model formulation to account for surface or atmospheric absorption are explained in Sect. 4.

Ground reflection is considered non-absorbing for two reasons: 


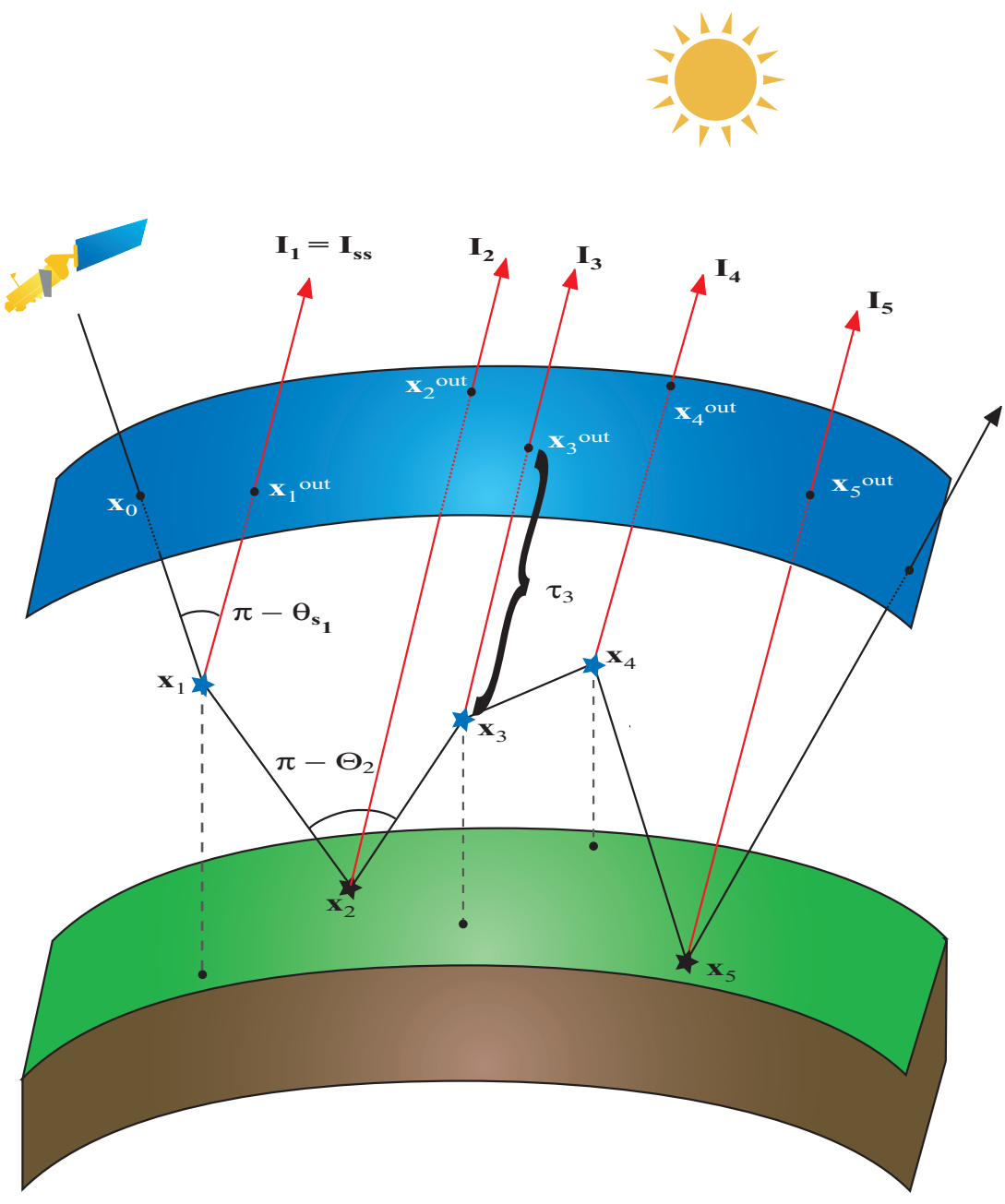

Fig. 1. Illustration of the backward Monte Carlo calculation. The photon leaves the satellite, enters the atmosphere at position $x_{0}$, is scattered at points $\boldsymbol{x}_{1}, \boldsymbol{x}_{3}, \boldsymbol{x}_{4}$ in the atmosphere (blue stars) and is scattered at points $\boldsymbol{x}_{2}, \boldsymbol{x}_{5}$ on the ground (black stars). After these 5 scattering events it ends its trajectory leaving the atmosphere. As an example the supplement of the scattering angle $\Theta_{2}$ is plotted. The relation between the old direction, the scattering angles and the new direction is explained in the Appendix in Sect. A3.5. At each scattering position the probability is calculated that the new direction after scattering would be towards the sun. The supplement of the angle used in Eq. (A26) is plotted for the first scattering event with the symbol $\pi-\theta_{\mathrm{s}_{1}}$. The points $x_{i}^{\text {out }}$ represent the exit positions of the photons if they would leave the atmosphere at the $i$-th scattering event in the direction of the sun. The radiance contributions are denoted as $I_{1} \ldots I_{5}$ (see also Eqs. A25 and 1). The first of these contributions is also used to calculate the radiance for single scattering $\left(I_{\mathrm{SS}}\right)$. The curly brace between $\boldsymbol{x}_{3}$ and $x_{3}^{\text {out }}$, marked with $\tau_{3}$ is an example of the trajectory used to calculate $\tau_{\mathrm{sca}}$ in Eq. (A27) and $\tau_{\text {ext }}$ in Eq. (2). The dashed lines represent the projections of the scattering points on the Earth surface.

1. we are evaluating only the ray-tracing part of the model. Absorption (both by molecules and the surface) will be considered later (see Sect. 4);

2. absorption will be computed, anyhow, by reducing the weight of the photons reflected by the surface (see Sect. 4).

Since the algorithm is formulated as a function of Earth radius, the first validation of the ray-tracing was performed by increasing the radius of McSCIA to $1000 \cdot R_{\text {earth }}$ and comparing our results with the Doubling and Adding model of the KNMI (de Haan et al., 1987; Stammes, 2001), a planeparallel RT model. The comparison was satisfactory, giving results with differences less than the statistical error of McSCIA $(0.1 \%)$.

Next, as in Oikarinen et al. (1999), we compared McSCIA results with results from Adams and Kattawar (1978) and Kattawar and Adams (1978) to verify the implementation of the spherical geometry. For the comparison, the Earth radius was set to $6371 \mathrm{~km}$, and the atmosphere was considered as a homogeneous spherical shell with a height of $100 \mathrm{~km}$. 


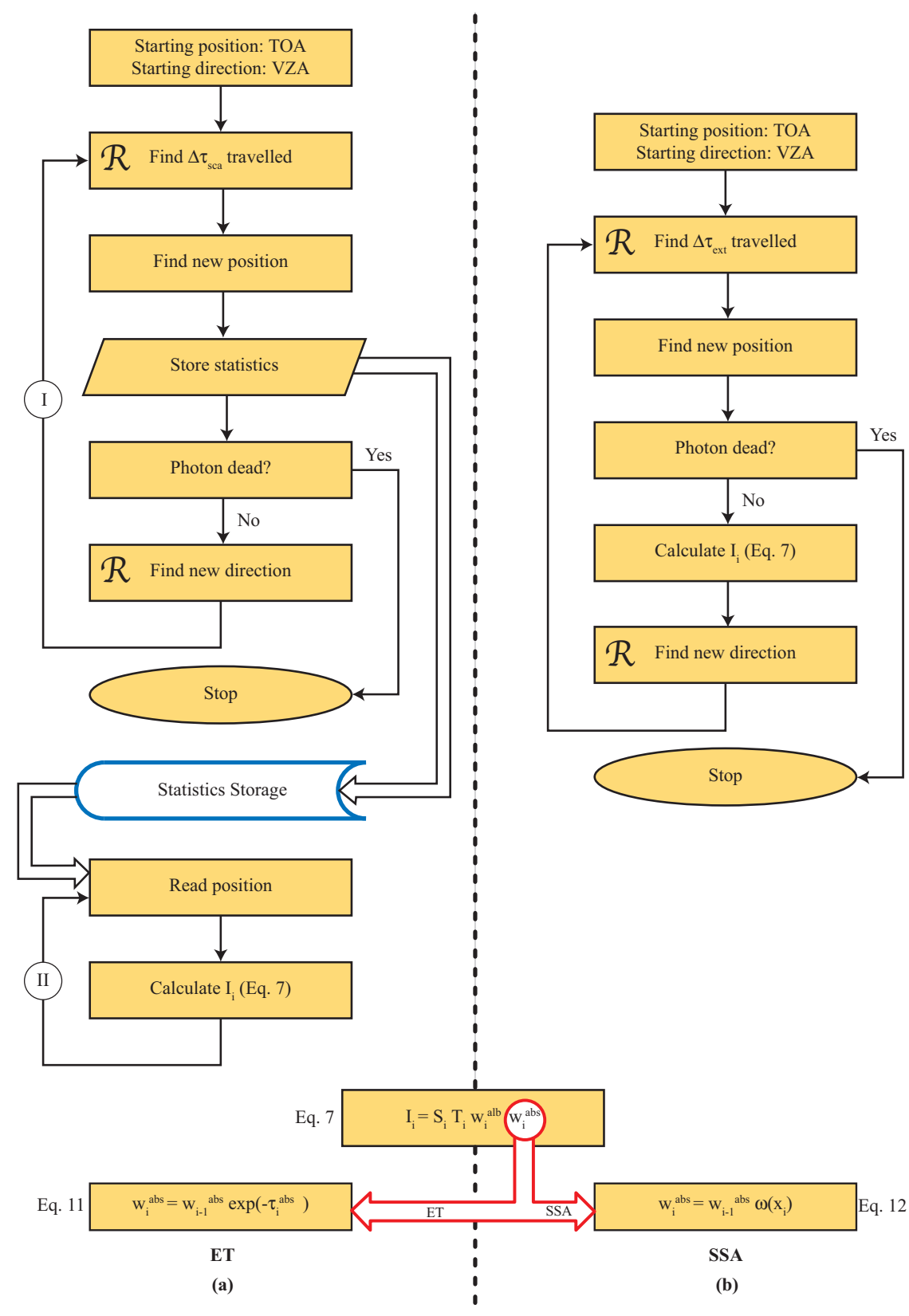

Fig. 2. Photon "life cycle" algorithm of one photon for the ET (a) and SSA (b) approaches. The boxes with a big $\mathcal{R}$ indicate the use of random numbers. Note that if absorption is considered, the methods use a different vertical coordinate: the ET method uses a scattering-only coordinate $\left(\tau_{\text {sca }}\right)$ while the SSA method uses the total extinction $\left(\tau_{\text {ext }}\right) . S_{i}$ is calculated with Eq. (A26) while the transmission factor $T_{i}$ uses Eq. (2). The albedo weight is calculated using Eq. (4). The lowest two boxes show another major difference in the radiance calculation: the ET method uses an exponential decrease (Eq. 5) while the SSA method uses the single scattering albedo (Eq. 6). While the SSA approach calculate the absorption in-line, the ET approach calculate it off-line. First the statistics is calculated by the first part (ray-tracing) of the model (I) and stored, then the second part of the model (II) calculate the absorption accessing the stored statistics.

The atmosphere was assumed to be conservatively scattering and the ground albedo was set to zero. In this case the photons end its trajectory when they touch the Earth's surface. The satellite viewing zenith angle $\theta_{\text {sat }}$ at TOA was varied between 0 and $88^{\circ}$ in the principal plane. This plane is de- fined by the relative azimuth angle values $\phi=\phi_{\text {sun }}-\phi_{\text {sat }}=0^{\circ}$ or $\phi=\phi_{\text {sun }}-\phi_{\text {sat }}=180^{\circ}, \phi_{\text {sat }}$ is assumed to be 0 . In the first case the setup looks like the one in Fig. 3, while in the latter the sun is in the left quadrant behind the satellite. As in Adams and Kattawar (1978), five values have been chosen 


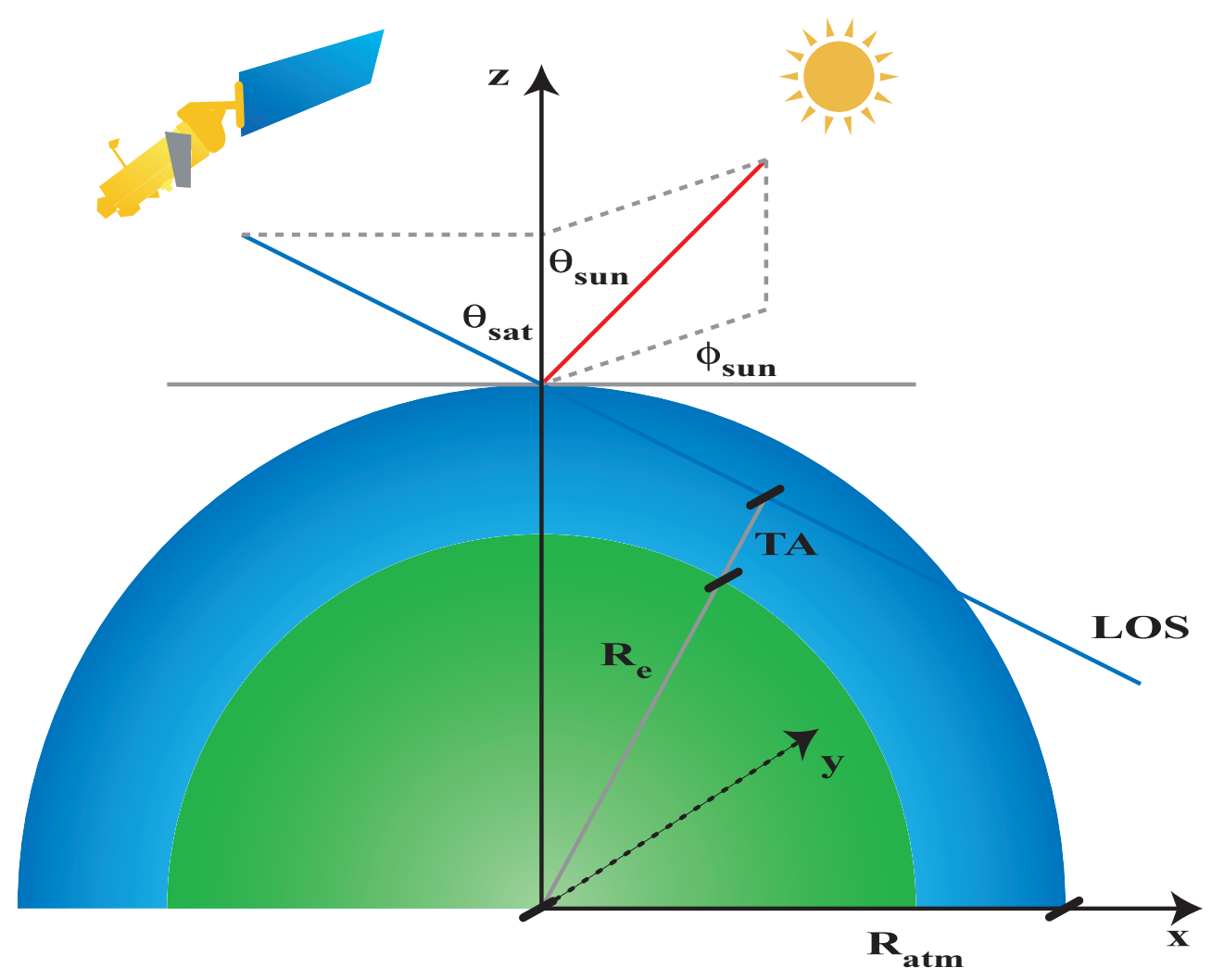

Fig. 3. Global coordinate reference system employed in McSCIA. The figure represents the satellite viewing zenith angle $\theta_{\text {sat }}$ The satellite viewing azimuth angle $\phi_{\text {sat }}$ is chosen to be zero, thus for the relative azimuth angle holds $\phi=\phi_{\text {sun }}-\phi_{\text {sat }}=\phi_{\text {sun }}$. In case of limb view, the tangent altitude TA is a function of the earth radius $\mathrm{R}_{\mathrm{e}}$ and the satellite viewing angle. The atmospheric radius $R_{\mathrm{atm}}$ is defined from the Earth centre to the top of atmosphere. The line of sight (LOS) is also depicted.

for the solar zenith angle $\theta_{\text {sun }}$ and azimuth angle $\phi_{\text {sun }}$ (see Table 1 and Fig. 4).

As an example of the comparison we show the results for two Rayleigh scattering atmospheres of optical thickness 0.25 . In the top panel of Fig. 5 we show the normalised single scattering (SS) radiance values, while in the bottom panel the normalised multiple scattering (TS) radiance values at top of atmosphere (TOA) for all the three solar positions.

The results of our simulations agree very well with the radiance values obtained by Adams and Kattawar (1978). In most cases results agree to the last digit given in the papers. Otherwise differences are normally smaller than $1 \%$. In some cases differences amount to $3-5 \%$. Since in the original papers there is no indication of the statistical error or of the number of photons used, the differences can be due to poorer statistics of the old models.

Figure 5 shows that we can capture several important features for the case of optical thickness of 0.25 . First of all, the dotted and dashed curves are not symmetric due to the solar position, that is not on nadir. For the case in which the solar zenith angle is $84.26^{\circ}$ the incoming solar radiation doesn't intersect the earth anymore, and the satellite can see
Table 1. Angles used for the comparison of Fig. 5 as defined in Adams and Kattawar (1978) and Kattawar and Adams (1978). The intercomparison geometries are also illustrated in Fig. 4.

\begin{tabular}{cccccc}
\hline$\theta_{\text {sun }}$ & $0^{\circ}$ & $70^{\circ}$ & $70^{\circ}$ & $84^{\circ}$ & $84^{\circ}$ \\
$\phi_{\text {sun }}$ & $0^{\circ}$ & $0^{\circ}$ & $180^{\circ}$ & $0^{\circ}$ & $180^{\circ}$ \\
\hline
\end{tabular}

also some part of ground beyond the terminator. This effect increases the difference between the radiance values for $\phi=0^{\circ}$ and $\phi=180^{\circ}$. Another important feature that McSCIA reproduces is that the radiance values increase until $80^{\circ}$ and decrease for greater angles. This feature it is characteristic of the spherical geometry: in this scenario after $80^{\circ}$ a planeparallel model would have only a monotonous increase of the radiance for increasing VZA (see Adams and Kattawar, 1978).

These findings are also similar to those obtained by the SIRO model (Oikarinen et al., 1999). Therefore, we are confident that our spherical implementation is correct. 


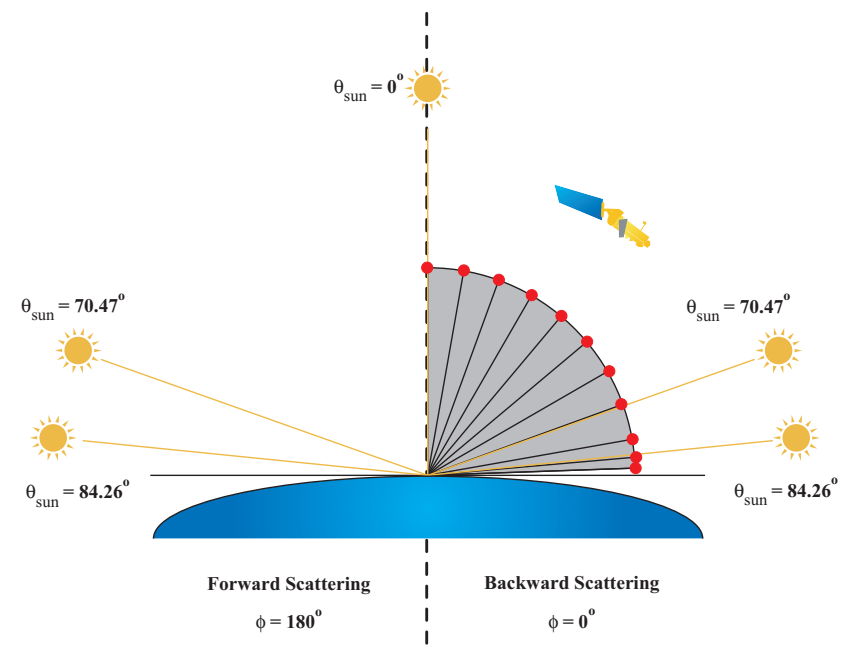

Fig. 4. Geometries used for the comparison with Adams and Kattawar (1978). The position of the sun (sun symbols) and of the satellite (red bullets) are indicated. See also Table 1.

\section{Absorption: Equivalence Theorem and Single Scat- tering Albedo}

The Equivalence Theorem (ET) of Irvine (1964) is a powerful way to include absorption in RT models. As discussed by van de Hulst (1980), the ET states that it does not matter whether the constituents doing the scattering and those doing absorption are identical. This means that if we distinguish two atmospheric constituents

- haze, that causes conservative scattering,

- gas, that causes absorption along the path between scattering points;

we can decide to treat absorption as if it would occur

1. only at the scattering points, using a single scattering albedo $(\omega)$ of the haze particles less than one, or

2. only along the path between scattering points with conservative scattering, using an exponential decrease of the radiance $I$ along the trajectory following LambertBeers law.

From now on we will call case 1 the SSA approach, and case 2 the ET approach.

4.1 McSCIA in a 3-D spherical atmosphere with absorption

In the book of van de Hulst (1980), it is spelled out how to use the ET for one layer, while in the work of Feigelson (1984) and Partain et al. (2000) possible uses of it for a multilayer geometry are explored. Partain goes as far as applying it to a case with a vertical profile of an absorbing trace gas. However, these applications are not suitable for a full 3-D study case. So we decided to explore a solution that could work in this case, as proposed, but not used, by O'Hirok and Gautier (1998). Nevertheless, the current implementation of the model is still bounded to a spherical shell atmosphere.

In McSCIA the atmosphere is formed by homogeneous spherical shell layers defined by the scattering coefficient $k_{\text {sca }}$, the absorption coefficient $k_{\text {abs }}$, the phase function $P(\mu)$ and the geometrical extension of each layer.

Using the ET approach (see the flowchart Fig. 2a), we perform a simulation of the model in a scattering-only atmosphere with ground albedo equal to 1 , as described in Sect. 2, and we store all the scattering and ground reflection positions. Once this scattering-only case has been computed, the contribution of each scattering event to the radiance can be evaluated, using a combination of the local estimate and weight techniques (e.g. see Marchuk et al., 1980; Davis et al., 1985; Marshak and Davis, 2005). If we follow only the j-th photon, similar to Eq. (A25), the value for the i-th event is given by:

$I_{i}=S_{i} \cdot T_{i} \cdot w_{i}^{\mathrm{alb}} \cdot w_{i}^{\mathrm{abs}}$

where $S_{i}$ is the scattering probability towards the sun defined in Eq. (A26). The other quantities are defined below.

The transmittance from the photon position to the sun is

$T_{i}=e^{-\tau_{\mathrm{ext}_{\mathrm{i}}}}$

where $\tau_{\text {ext }}$ is the optical thickness travelled by the photon from its current position to the sun, calculated integrating $k_{\text {ext }}$ along the photon path $s$

$\tau_{\mathrm{ext}_{\mathrm{i}}}=\int_{s} k_{\mathrm{ext}}(\boldsymbol{s}) \mathrm{d} \boldsymbol{s}=\int_{s}\left(k_{\mathrm{sca}}(\boldsymbol{s})+k_{\mathrm{abs}}(\boldsymbol{s})\right) \mathrm{d} \boldsymbol{s}$.

The difference between Eq. (2) and Eq. (A27) is that now also absorption is included in the transmission, as can be seen by the difference between Eq. (3) and Eq. (A28). In the ET method this factor is calculated off-line, after the ray-tracing.

To account for a surface albedo less than unity, the weight due to surface reflections, $w_{i}^{\mathrm{alb}}$, is the cumulative ground albedo at position $\boldsymbol{x}_{i}$

$w_{i}^{\mathrm{alb}}=\prod_{k=1}^{i} \alpha\left(\boldsymbol{x}_{k}\right)$.

The coefficient $\alpha\left(\boldsymbol{x}_{k}\right)=1$ if $\boldsymbol{x}_{k}$ is in the atmosphere, and $\alpha\left(\boldsymbol{x}_{k}\right)=a\left(\boldsymbol{x}_{k}\right)$ if $\boldsymbol{x}_{k}$ is on the Earth surface, with $a\left(\boldsymbol{x}_{k}\right)$ the ground albedo at point $\boldsymbol{x}_{k}$. With this approach the effects of a $2-D$ variable ground albedo can be easily evaluated.

The cumulative atmospheric absorption weight, $w_{i}^{\mathrm{abs}}$, is the product of the transmission function from point to point

$w_{i}^{\mathrm{abs}}=\prod_{k=1, i} e^{-\tau_{\mathrm{abs}} \mathrm{k}}$ 

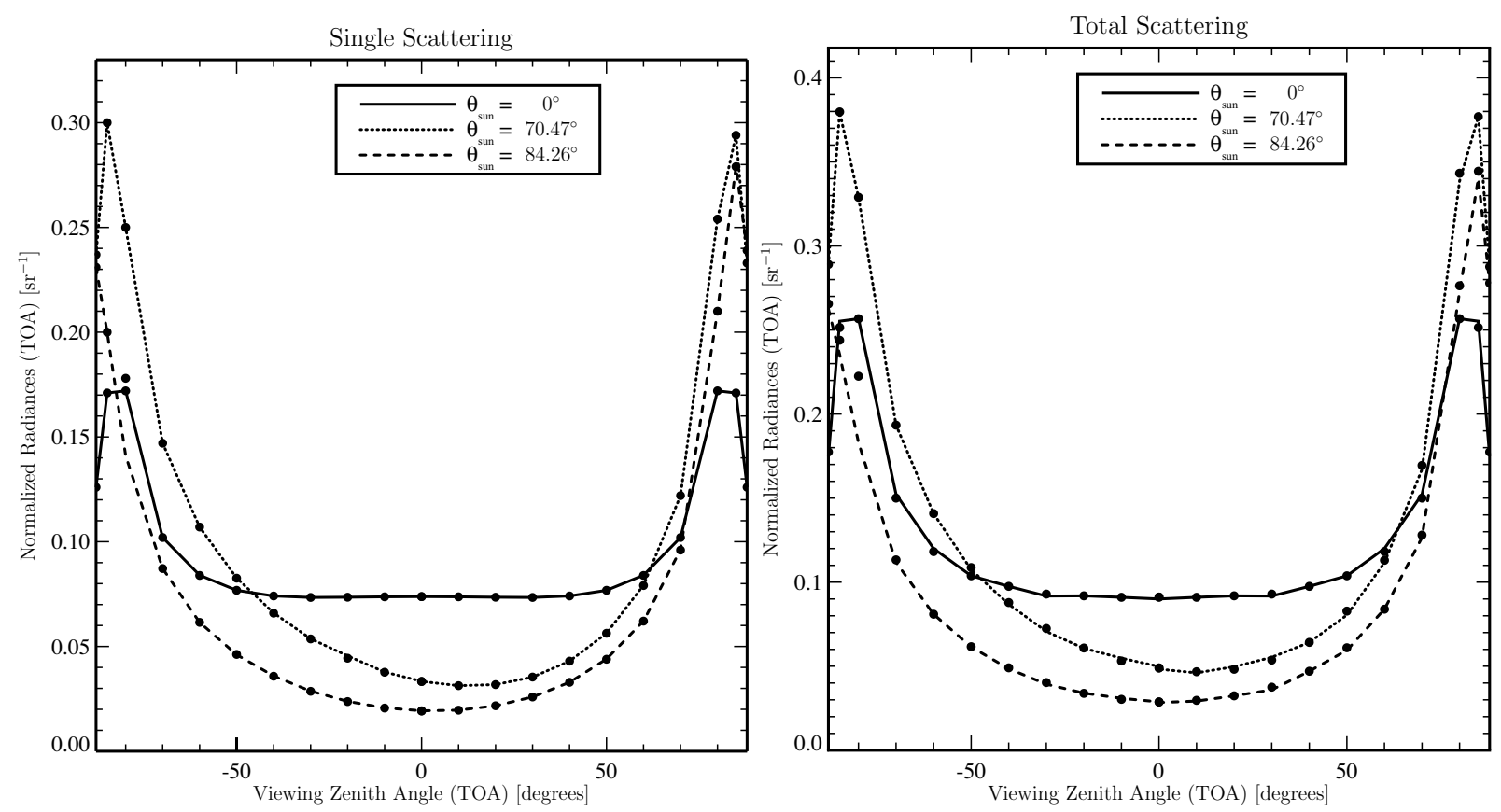

Fig. 5. Comparison between McSCIA results (bullets) and those of Adams and Kattawar (1978) (lines). The normalised radiance for an incoming solar flux of $\pi$ as a function of the satellite viewing angle at TOA $(101 \mathrm{~km})$ is plotted for three different solar zenith angles. The view angle scan is done through the principal plane, $\phi=0^{\circ}$ (right part of the figures, VZA $>0$ ) and $\phi=180^{\circ}$ (left part of the figures, VZA $<0$ ). The values of the angles used, are in Table 1 as illustrated by Fig. 4. The atmosphere is a homogeneous, conservative, Rayleigh scattering layer with optical thickness of 0.25 . Top panel: single scattering. Bottom panel: total scattering.

where $\tau_{\mathrm{abs}}=\int_{s} k_{\mathrm{abs}}(s) \mathrm{d} s$ and $s$ is the line connecting $\boldsymbol{x}_{k-1}$ and $\boldsymbol{x}_{k}$.

In the ET approach the atmospheric absorption is accounted for in calculating the extinction from the scattering position towards the sun (Eqs. 2, 3) and from scattering point to scattering point (Eq. 5). Both these factors are calculated off-line.

If we use, instead, the SSA algorithm to account for absorption, we follow the flowchart of Fig. 2b. Now the scattering of the photons is calculated in a scattering and absorbing atmosphere. The contribution of each scattering event is given, like for the ET case, by Eq. (1), but this time the absorption term $w_{i}^{\text {abs }}$ is given by

$w_{i}^{\mathrm{abs}}=\prod_{k=1, i} \omega\left(\boldsymbol{x}_{k}\right)$

Inspecting the flowcharts of Fig. 2 it is easy to see that the absorption is calculated "off-line" for the ET method (a), while it is calculated "on-line" for the SSA case (b). Thus, it is clear that the results of the SSA simulations cannot be re-used if we change the absorption properties of the atmosphere. We should in this case also re-calculate all the scattering positions, since they depend on the absorption coefficients in the 3-D atmosphere. However, the ET calculation with a scattering-only atmosphere can be applied to any distribu- tion of absorbers, as long as the scattering properties remain unchanged.

Now that the differences between the two approaches of calculating absorption have been spelled out, we will show that they give equivalent results with a simple 1-D MC RT model.

\subsection{Simple 1-D demonstration of the Equivalence Theorem}

To demonstrate the use of the ET we have developed a simple one-dimensional MCRT model. The only aim of this model is to illustrate the ET, and would be ideal as a classroom example for RT. It is fully described in Appendix B and it can perform simulations in both SSA and ET methods.

To compare the level of agreement between the two methods many scenarios were simulated (see Table B1 and Fig. B1). The basic scenario was that of Rayleigh scattering with an ozone-like absorber. The absorption peak altitude was always $z_{\max }=22 \mathrm{~km}$. In each of these scenarios, the amount of absorption was varied in depth $(d)$ and absorption peak value $\left(c_{a}\right)$ (see Table B1) giving more that three thousand different scenarios, with absorption optical thickness ranging from 0 to 7.4 and scattering optical depth ranging from 0.08 to 8.0 .

To evaluate the statistical error $(\sigma) 10$ simulations, each with $10^{5}$ photons, were performed. The average of these 10 


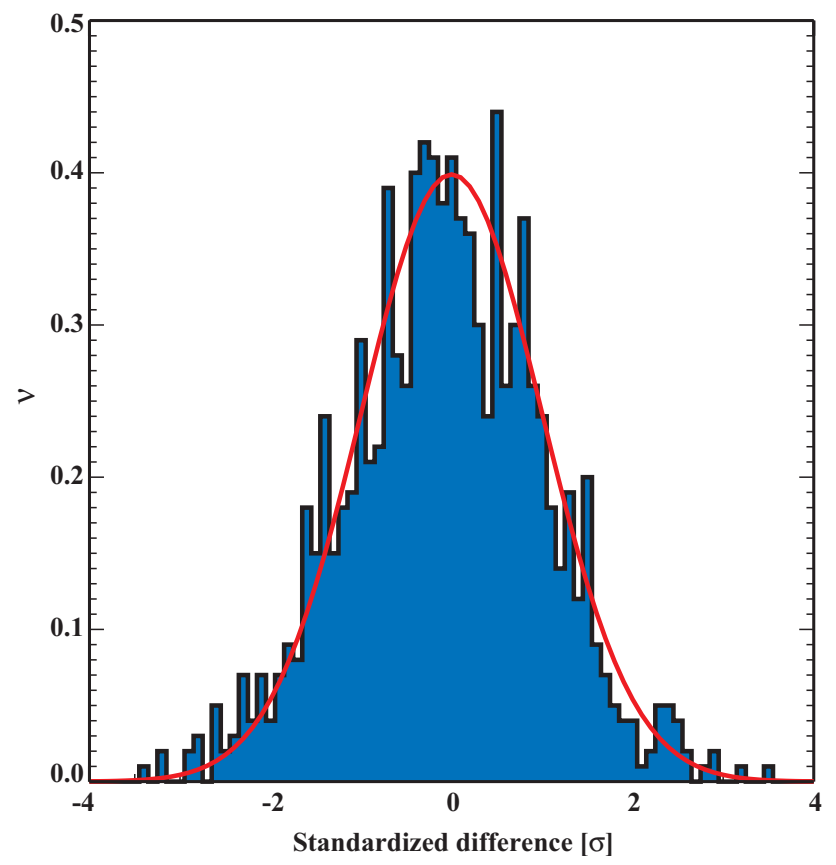

Fig. 6. Frequency distribution of the standardised difference (Eq. 8) between the SSA and ET approaches for the simple 1-D model. Scenarios are listed in Table B1. The data are well approximated by a standardised normal distribution. The results are for a thousand simulations of one million photons each.

intensities represents the radiance and the sample standard deviation is used to estimate the spread of the radiance. The error is, thus, calculated via the formula

err $=\sigma / \bar{I} \cdot 100 \quad[\%]$.

Then, the results of the ET $\left(I_{1} \pm \sigma_{1}\right)$ and SSA $\left(I_{2} \pm \sigma_{2}\right)$ cases were compared calculating the standardised difference $\left(\Delta_{S}\right)$ between the two models

$\Delta_{S}=\frac{I_{1}-I_{2}}{\sqrt{\sigma_{1}^{2}+\sigma_{2}^{2}}}$.

Figure 6 shows the $\Delta_{S}$ values of the different realisations described by Table B1. The differences are well approximated by a normal distribution and the agreement between the two models does not seem to be related to the optical thickness. Thus, we can conclude that the two different approaches give statistically equivalent results and that the remaining differences are caused by statistical fluctuations, that are an intrinsic part of every MC process.

\section{Performance of McSCIA in 3-D}

\subsection{Validation of McSCIA in 3-D}

The validation of McSCIA in a full 3-D case was performed by a comparison to the results of a MC reference model described in Loughman et al. (2004).
Table 2. Cross section used for the comparison between McSCIA and MCC++ (from Loughman et al., 2004).

\begin{tabular}{lccc}
\hline & & \multicolumn{2}{c}{ Wavelength $[\mathrm{nm}]$} \\
& & 325 & 345 \\
\hline & & & \\
Rayleigh scattering cross section & $10^{-25}\left[\mathrm{~cm}^{2}\right]$ & 0.4022 & 0.3120 \\
Ozone absorption cross section & $10^{-20}\left[\mathrm{~cm}^{2}\right]$ & 1.451 & 0 \\
\hline
\end{tabular}

The agreement between two MC models depends critically on the way the optical properties of the atmosphere are discretised (Postylyakov et al., 2003). The model MCC++ (Postylyakov, 2004) was chosen as a reference model since it uses a piece-wise constant distribution function with discontinuities at grid points which the same as in our implementation. As outlined by Postylyakov et al. (2003), differences between the models up to $1 \%$ are acceptable since the optical properties are derived in different ways. However, while the atmospheric layering difference may not be the dominant source of differences, during the intercomparison of McSCIA with the model of $\mathrm{H}$. Walter (Walter et al., 2006), we have noticed that a small difference in the pointing of the satellite, along with slightly different optical properties of the atmospheres, could cause differences of more than $1 \%$.

We compared with the first case of Loughman et al. (2004, Sect. 3.2, Fig. 4): a limb scan in an aerosol free atmosphere, for $\lambda=345 \mathrm{~nm}$ and $\lambda=325 \mathrm{~nm}$. We use the MODTRAN (Berk et al., 1989) tropical atmospheric density and $\mathrm{O}_{3}$ profiles, with the cross section for Ozone and Rayleigh provided in the Loughman et al. (2004) (see also Table 2). The vertical profiles of $k_{\text {sca }}$ and $k_{\text {ext }}$ are shown in Fig. 7.

The atmosphere was discretized in 100 homogeneous layers equally spaced ( $1 \mathrm{~km}$ depth each), with the Earth radius set to $6377.640 \mathrm{~km}$. For scattering the Rayleigh phase function was used. Polarisation was neglected (scalar case).

The results of the intercomparison are shown in Figs. 8 and 9 for $345 \mathrm{~nm}$ and $325 \mathrm{~nm}$, respectively, both for single scattering and total scattering, using the ET method.

The percentage difference between the two models is defined as

$\delta_{\mathrm{MM}}=100 \cdot\left(I_{\mathrm{McSCIA}}-I_{\mathrm{MCC}++}\right) / I_{\mathrm{MCC}++}$

The standard deviation $\sigma_{\mathrm{MM}}$ of the comparison is defined as

$\sigma_{\mathrm{MM}}=\sqrt{\sigma_{\mathrm{McSCIA}}^{2}+\sigma_{\mathrm{MCC}++}^{2}}$.

Generally the agreement between the two models is within two $\sigma_{\mathrm{MM}}$ (shaded region) and is better without $(345 \mathrm{~nm})$ than with $(325 \mathrm{~nm}) \mathrm{O}_{3}$ absorption. The agreement is worse in the upper part of the scan than in the lower part for the case of $325 \mathrm{~nm}$.

We think that these features have a common cause: we had to generate our own optical atmosphere for the Loughman et al. 


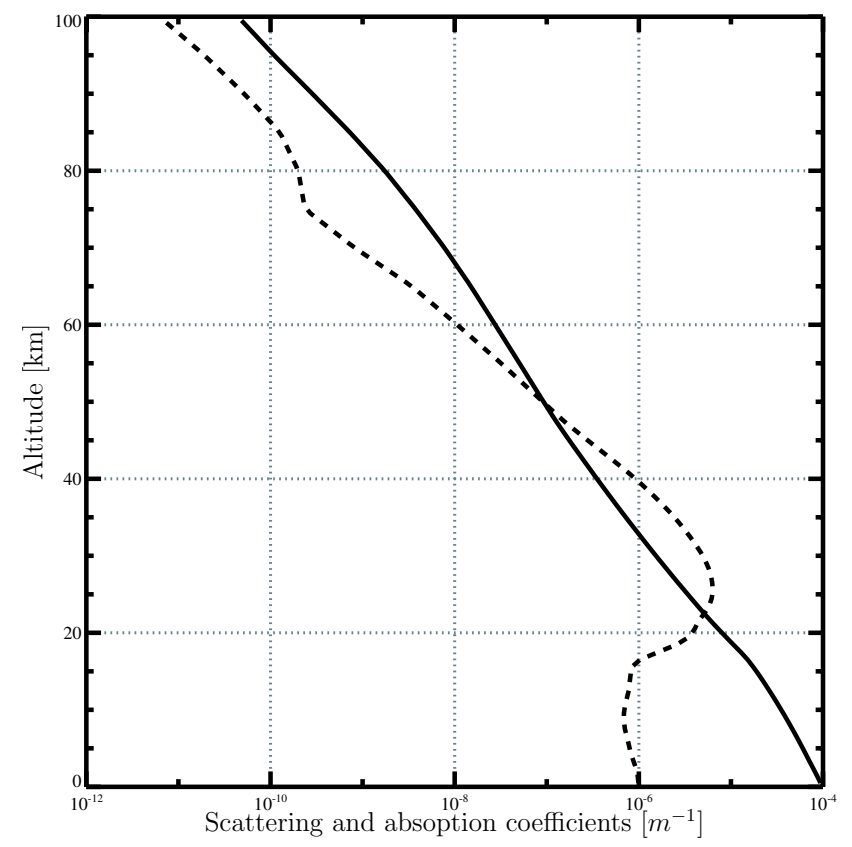

Fig. 7. Vertical profile of the Rayleigh scattering coefficient (full line) and ozone absorption coefficient (dashed line) at $325 \mathrm{~nm}$. The scattering coefficient profile for $345 \mathrm{~nm}$ is identical to the $325 \mathrm{~nm}$ profile but scaled by a factor 0.77573347 , whereas ozone absorption is assumed to be negligible at $345 \mathrm{~nm}$.

(2004) intercomparison c ase, so that some small differences exist between the two model set-ups. Another issue is that the geometries used in McSCIA and in the intercomparison paper are not the same: we use angles at the top of the atmosphere instead of angles at the tangent altitude, and small numerical errors can be introduced in the conversion. The differences between the two models are well under $0.2 \%$ for most cases and always smaller than $0.5 \%$.

In conclusion we are confident that we correctly implemented absorption and the spherical geometry in our McSCIA model, and we have already a proof that the ET method gives good results.

\subsection{Comparison between ET and SSA in 3-D}

Here we want to investigate whether the ET approach gives equivalent results as the SSA approach in 3-D for a wide range of scenarios with different absorption profiles. In particular we want to analyse extreme cases from very thin to very thick atmospheres for tangent altitudes from 20 to $60 \mathrm{~km}$, descibed in Table 3. McSCIA simulations were performed with 1 million photons and the atmospheric profiles were generated with Eq. (B1) using the parameter values of Table 3.

As can be seen from Fig. 10 the ET and SSA results agree very well. The standardised differences between the two ap-
Table 3. Parameters for Eq. (B1) used to generate the atmospheric profiles for the scenarios ET/SSA comparison for the 3-D case. The total scattering optical thickness is about 0.8 . The total absorption optical thickness varies from 0 to 7.4.

\begin{tabular}{cl}
\hline$z_{\text {scale }}$ & $8.0 \mathrm{~km}$ \\
$z_{\max }$ & $22.0 \mathrm{~km}$ \\
\hline$c_{S}$ & $0.1 \mathrm{~km}^{-1}$ \\
$c_{a}$ & $0.0001,0.001,0.01,0.05,0.1,0.2,0.3 \mathrm{~km}^{-1}$ \\
$d$ & $0.1,1.0,5.0,10.0 \mathrm{~km}$ \\
\hline
\end{tabular}

proaches are rarely larger than $0.5 \sigma$, and always smaller than one $\sigma$. More importantly, Fig. 10 shows a tendency towards a normal distribution of SD, like in Fig. 6. However, in 3$\mathrm{D}$ the computation time is much longer than in 1-D, so we could not make a similar number of runs as was made in the 1-D case.

Both approaches give the same results for different scenarios with a variable amount of absorber, concentrated in one thin layer or spread over many layers. With McSCIA it is now possible to study several 3-D absorption scenarios with one simulation, thanks to the power of the Equivalence theorem.

\section{A 3-D example: sensitivity of measured radiance}

A problem that is crucial at this point in history of satellite development, is determining where the radiation measured by a satellite is absorbed in the 3-D atmosphere. This is, in fact, strictly related to the maximum resolution that a space born instrument can achieve.

One approach to understand this problem, is to calculate the fraction of absorption that is occurring in the pixel measured by the satellite (black cone in Fig. 11). This can be efficiently calculated using the equivalence theorem in McSCIA to derive the sensitivity of each layer to changes in trace gas concentrations.

Having kept all the photon statistics, it is now possible to calculate this sensitivity by just applying the correction factor $\exp \left(-\tau_{p}\right)$ to Eq. (1), where $\tau_{p}$ is the optical thickness added to perturb the gas concentration. Moreover, from Eq. (1) it is clear that the code can also efficiently calculate these values as a function of ground albedo.

Table 4 gives the fraction of absorption that is taking place inside the pixel at $1 \mathrm{~km}$ and $30 \mathrm{~km}$ of altitude, and for ground albedo $\mathrm{a}=0.05$ and $\mathrm{a}=1.0$. The results are calculated using the same atmospheric profile as described in Fig. 7, for $325 \mathrm{~nm}$. The satellite is looking in nadir with a circular ground pixel of $24 \mathrm{~km}$ of diameter, while the solar zenith angle is $40^{\circ}$ (see Fig. 11).

The values of Table 4 show that there is a smoothing effect and a geometrical effect. While the latter is mainly due to the 


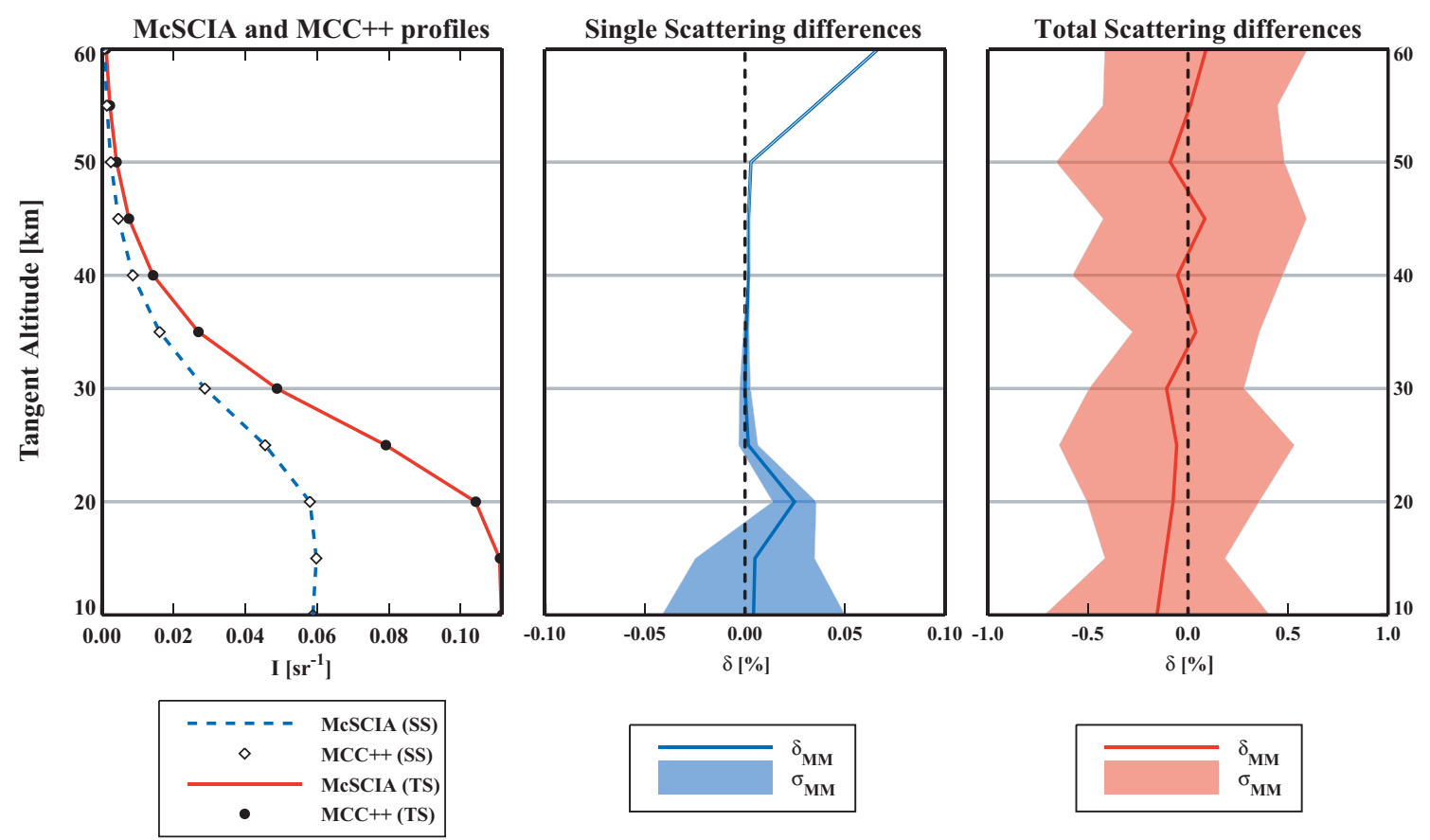

Fig. 8. Comparison of limb profiles of radiance $I$ between McSCIA (ET) and MCC ++ at $\lambda=345 \mathrm{~nm}$, for an aerosol-free atmosphere. The solar zenith angle is $39.2933^{\circ}$ and the relative azimuth is $111.746^{\circ}$ at LOS. In the left panel the radiance values of McSCIA (lines) and $\mathrm{MCC}++$ (filled circles and diamonds) are shown. In the centre panel the single scattering percentage difference $\delta_{\mathrm{MM}}(\mathrm{Eq} .9)$ is shown, with the error $\sigma_{\mathrm{MM}}$ (Eq. 10) represented by the shaded area and the $0 \%$ difference shown with a dashed line. In the right panel, $\delta_{\mathrm{MM}}$ is shown for total scattering. See also Loughman et al. (2004, Fig. 4).
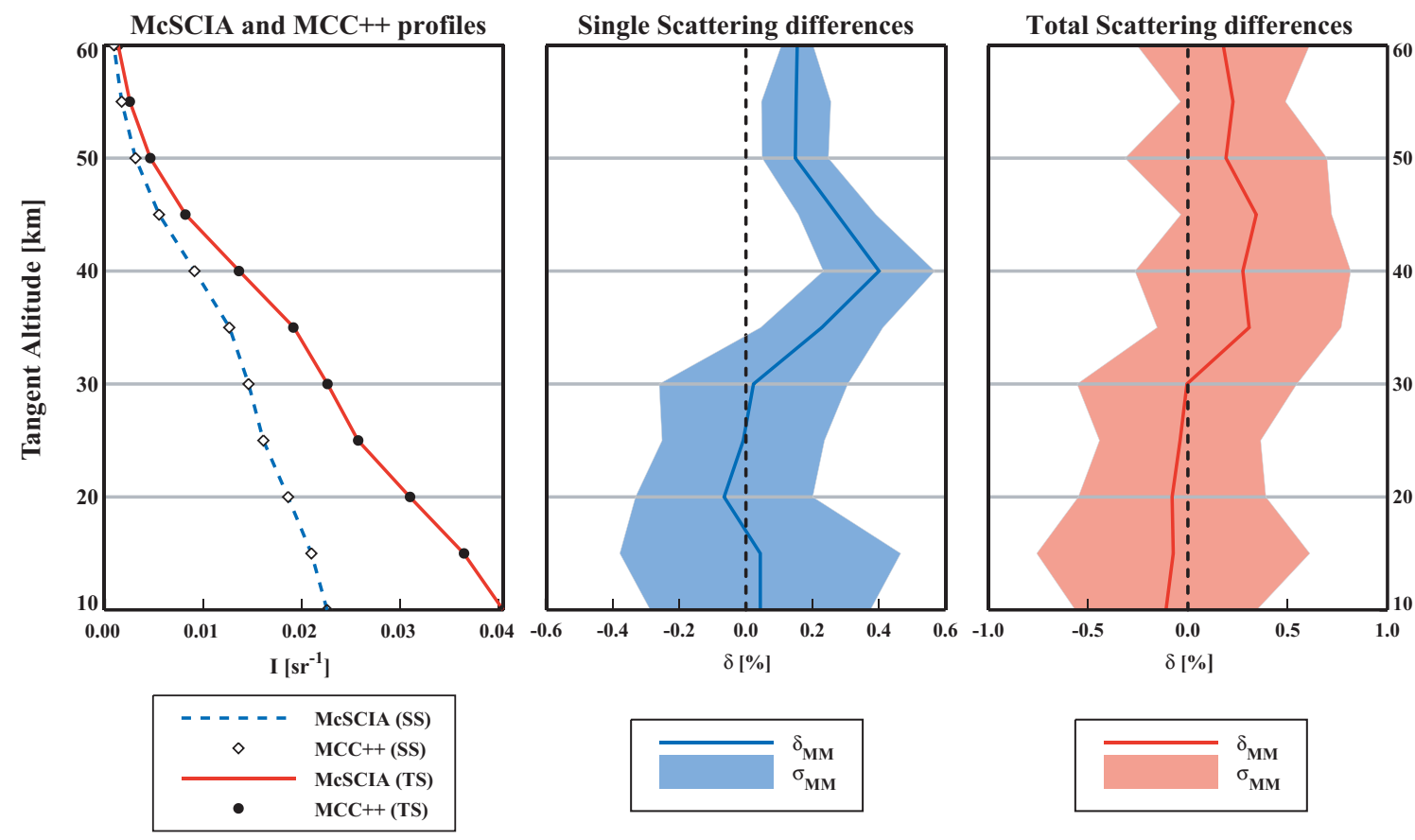

Fig. 9. Same as Fig. 8 but at $\lambda=325 \mathrm{~nm}$. 


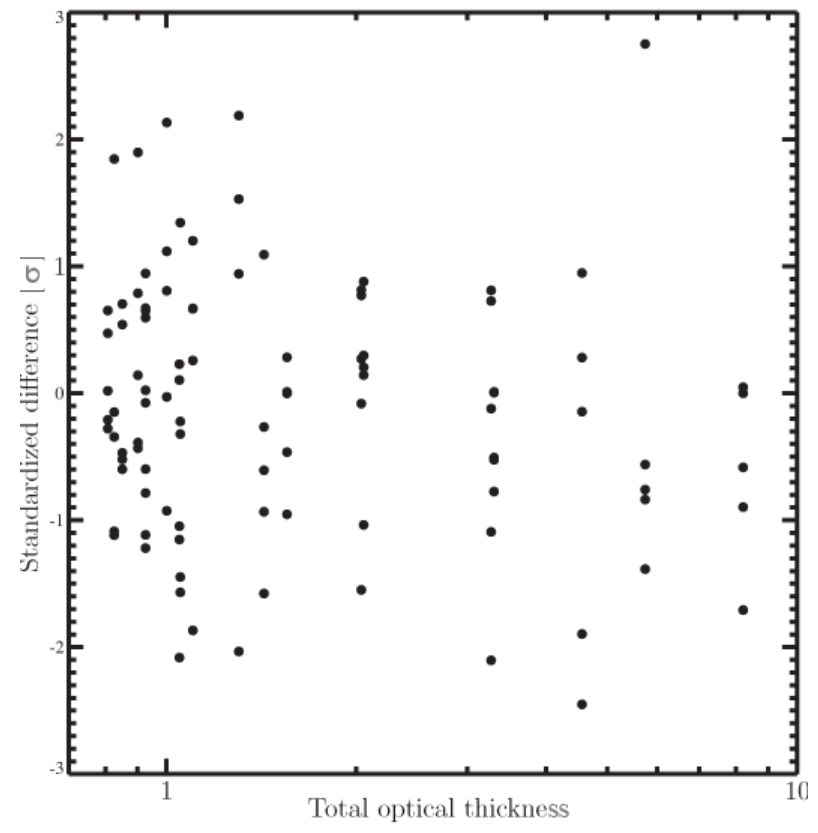

Fig. 10. Standardised difference (Eq. 8) between the SSA and ET of McSCIA (3-D), for the cases of Table 3 and tangent altitudes $20,30,40,50,60 \mathrm{~km}$. The simulations were performed with solar zenith angle $37^{\circ}$ and azimuth $101^{\circ}$.

geometry of the problem and to single scattering, the former is due to multiple Rayleigh scattering.

The single scattering photons follow a straight line sunscattering point-satellite that at some altitudes does not cross the satellite pixel (see red and black circle in Fig. 11). This geometrical effect is more important in the higher levels of atmosphere, as can also be seen by the differences between $1 \mathrm{~km}$ and $30 \mathrm{~km}$, especially in the case of no scattering atmosphere. In fact the two circles almost completely overlap on the $1 \mathrm{~km}$ layer, while they are completely separated at $30 \mathrm{~km}$ of altitude (the layer represented in Fig. 11).

The differences in fraction of absorption for the cases with ground albedos of 0.05 and 1.0 show clearly that the increase of multiple scattering decreases the fraction of absorption taking place in the pixel. However, the total amount of absorption taking place is larger for larger albedos.

If we compare the no scattering case with the $a=0.05$ case at $1 \mathrm{~km}$ of altitude, it is possible to see the smoothing effect due the Rayleigh scattering. This effect increases as the ground albedo increases. However, at $30 \mathrm{~km}$ we can see a strange behavior: the fraction of absorption is lower in the case without scattering than in the other two. This can be explained by the fact that the geometry that we chose is very similar to the one depicted in Fig. 11: the two circles (red and black) are just next to each other. In that case, without scattering, the photons would cross the level only through the two circles and the pixel would account for less than half of the

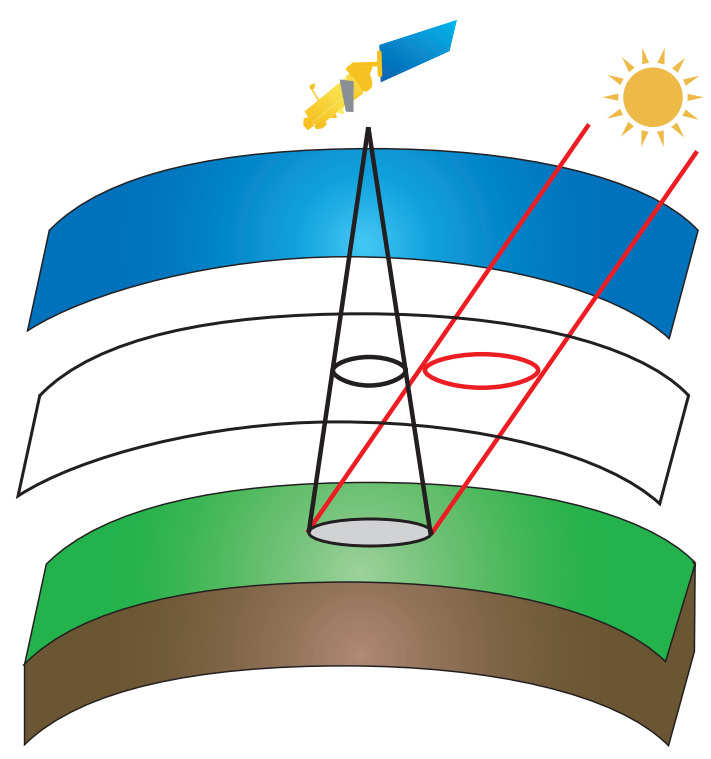

Fig. 11. Schematic illustration of the light paths of measured photons in a non scattering atmosphere (not at scale). The black cone represents the 3-D pixel observed by the satellite instrument. The black circle filled in gray represents the instrument ground pixel. The layer in which are depicted the two circles represents the geometrical setup for the case studied at $30 \mathrm{~km}$ of altitude. Note that on this layers the two circles do not overlap.

Table 4. Fraction of absorption inside the pixel. In the no scattering case the only processes taking place are absorption and ground reflection.

\begin{tabular}{lccc}
\hline & $\mathrm{a}=0.05$ & $\mathrm{a}=0.8$ & no scattering \\
\hline $1 \mathrm{~km}$ & $61 \%$ & $57 \%$ & $97 \%$ \\
$30 \mathrm{~km}$ & $51 \%$ & $48 \%$ & $43 \%$ \\
\hline
\end{tabular}

absorption (the other circle is bigger and the photons cross it with a slant path). However, if we consider an atmosphere with Rayleigh scattering, a lot of bright single scattering photons would cross in the pixel circle without crossing the other one.

These section shows how the ET in McSCIA can be used to speed up calculations in which we have 3-D variation of trace gas absorbers. With this method we are able to calculate the fraction of absorption that is occurring inside the pixel observed by a space born instrument. The results that we found show that this quantity is very important and that it is composed by a geometrical and a scattering part. 


\section{Discussion}

In this section we discuss different implementations of the ET used in literature and the one used in McSCIA.

The aim of this paper is to show the validity of the ET approach in 3-D RT problems. This power of the ET approach has been recognised earlier. For instance, van de Hulst (1980) states that RT can be defined in terms of inert parameters, i.e. variables that determine the radiance field but remain constant in many equivalent situations. These inert parameters may be clouds, geometrical setting, phase function, etc.

Considering an atmosphere in which the haze provides scattering and absorption and a gas provides absorption, the ET can be summarised by (van de Hulst, 1980, p. 576)

$$
\begin{aligned}
I(\omega, \tau, \gamma)= & \sum_{n} I_{n}(\tau, \gamma=0) \omega^{n} . \\
& \cdot \int_{0}^{\infty} p_{n}(\tau, \gamma=0, \lambda) e^{-\gamma \lambda} \mathrm{d} \lambda .
\end{aligned}
$$

where $\omega$ is the haze single scattering albedo, $\lambda$ the optical path-length and $\gamma$ is the ratio of the gas absorption to haze extinction. The equation tells that the radiance in an absorbing atmosphere $I(\omega, \gamma)$ can be calculated as a weighted sum over the number of scattering events $n$ of the radiance $I_{n}(\gamma=0)$ due to each scattering order calculated in a nonabsorbing atmosphere. The weight contains the absorption, which is calculated from the statistical part of the information in the form of the normalised probability distribution (pdf) of photon path-lengths $p_{n}(\gamma=0, \lambda)$, calculated for each scattering order. The important point here is that the pdf is calculated in an atmosphere without absorption and is subsequently used to calculate the absorption contribution.

In the work of Partain, Heidinger and Stephens (Partain et al., 2000; Stephens and Heidinger, 2000; Heidinger and Stephens, 2000, 2002) the ET, as expressed by Eq. (11), has been presented and employed, extending the original formulation of van de Hulst (1980). The use of geometrical instead of optical photon paths allow Partain et al. (2000) to extend the ET to multiple homogeneous layers. The ET is actually extended also in a way that takes into account ground albedo, the single scattering albedo and a vertical gas profile. The problem is solved by the introduction of a pdf that represents the statistics for gas, particle and ground absorption. Since storage of the complete pdf would make the model slower than performing SSA calculations, an approximate pdf is constructed. The authors recognised that such an approach introduces a bias, i.e. an overestimate of the spectral absorption.

For the work of Cahalan et al. (1994) holds similar consideration as for the one of Partain.

Another approach was introduced by Feigelson (1984). The concept of equivalent trajectories is used to condense the information of all the individual photon trajectories in an "average" trajectory. Basically this quantity represents the aver- age number of times that a model layer has been crossed vertically by the "average" photon. In principle, such an average photon path calculated for a scattering-only atmosphere might be convolved with different absorption profiles. However, it can be shown that this approach is only valid in the weak absorption limit (i.e. $\left.\exp \left(-\tau_{\mathrm{abs}}\right)=1-\tau_{\mathrm{abs}}\right)$. Apart from the approximate nature of these approaches, they suffer also from the limitation that they cannot be used for 3-D varying absorption features.

None of these implementations of the ET compared the use of the ET and the traditional SSA approach for a 3-D case. Thus, we extended the ideas outlined above by retaining all information of the scattering photons in a 3-D spherical atmosphere. Although such an approach is not efficient (storage of the scattering positions of $\approx 10^{6}$ photons) this approach allowed us to prove the validity of the ET. However, many possible improvements to this brute force approach can be considered. For example, modern (multi-processor) computers can handle these problems adequately for some selected scattering scenarios.

An issue that deserves improvement in McSCIA is the quality of the statistical information of photons. When atmospheric absorption is strongly dominant over scattering, simulations of a scattering-only atmosphere are not representative for the true situation. For instance, in a scattering-only case many photons will travel to the surface while in reality most of the photons would be subject to atmospheric absorption. A way to circumvent this problem is the use of a mixed SSA and ET approach. The conventional SSA method is used to simulate photon paths in an absorbing and scattering atmosphere (e.g. employing a standard absorption profile). Afterwards, 3-D absorption perturbations can be studied using the ET approach. Using this approach, the statistical photon path information that is stored represents the actual situation more efficiently.

A strong point of our implementation is the possibility of using a 2-D varying ground albedo in a simple way. This is due to the fact that the ground albedo only appears in Eq. (4); it is very simple to relate its value to an albedo map.

The gain of speed due to the use of the ET depends critically on how it is used. In fact, the gain of speed in McSCIA depends on the amount of statistical information retained after the ray-tracing part. With a small amount of information retained it would be possible to build a very fast model but without too much flexibility (e.g. as done by Partain et al., 2000). Otherwise, if more information would be saved, in order to study more complex cases (e.g. 2-D absorber fields (Spada et al., 2006 $\left.{ }^{1}\right)$ ), then the gain of speed would be less.

Partain et al. (2000) retained the statistical information as a PDF. This condensed form of information works efficiently, but is only an approximation. So, we decided to retain all ray-tracing information. At the moment the ray tracing part

\footnotetext{
${ }^{1}$ Spada, F., Krol, M. C., and Landgraf, J.: 3D weighting functions and radiative smoothing, in preparation, 2006.
} 
of the model is written in FORTRAN-90 and all statistical information is stored (scattering position, scattering angles, photon weight). A post-processing code is written in Interactive Data Language (IDL) which provides good visualization tools. From this choice it is clear that computing speed is not a consideration at the moment.

The traditional way to use the ET is to employ the fact that scattering varies much less with wavelength than absorption, especially in spectral windows with sharp absorption lines. This allow a fast calculation of absorption lines under the assumption that scattering is constant. However, this is not the only way in which it can be used.

While the agreement between the codes in the I3RC (Cahalan and Davies, 2000) shows that the ET works, we felt it necessary to demonstrate how well it can perform. The fact that in McSCIA we have statistically identical results, while there is a biasing in the Partain et al. (2000) case, shows that not every implementation of the ET is the same and that people should be aware of it when they employ the ET in their model.

After having demonstrated in this paper for the first time the validity of the ET in a spherical 3-D environment, we have shown, in Sect. 5.2, an example of calculation of the fraction of absorption occurring in the pixel seen by the satellite. We want to emphasize that these results depend critically on the wavelength used, on the geometry of the observation, on the ground albedo on the atmospheric setup (clouds, aerosols, ....).

We are currently employing the ET to study the sensitivity of the TOA radiance for 3-D variations of absorption in the atmosphere (Spada and Krol, 2005), by calculating 3-D weighting functions. This is relevant to e.g. satellite measurement of tropospheric pollution.

\section{Summary and conclusions}

A Monte Carlo adjoint RT model, named McSCIA, has been developed. It is intended for the study of UV-vis-NIR RT problems in a fully 3-D spherical atmosphere, e.g. to study the radiances measured by limb-viewing instruments like SCIAMACHY. McSCIA can use mixed Rayleigh and Henyey-Greenstein phase functions and can employ a 2-D varying Lambertian surface reflection. Refraction and polarisation are not included.

Results from the ray-tracing module compare well with published results for non-absorbing plane-parallel cases, for different phase functions (Rayleigh and Henyey-Greenstein) and several nadir geometries and sun positions.

The spherical implementation of McSCIA was successfully validated against the state-of-the-art Monte Carlo model MCC++ (Postylyakov, 2004) using a spherical shells atmosphere, as described in Loughman et al. (2004), and earlier results (Adams and Kattawar, 1978; Kattawar and Adams,
1978) simulating a homogeneous spherical single shell atmosphere, as described in their paper.

In McSCIA the absorption has been implemented using two different methods. The traditional SSA methods which uses the scattering and absorption optical depth as vertical coordinate and employs the single scattering albedo $\omega$ at the simulated scattering positions to take into account absorption of radiation. The ET approach which simulates photons in a scattering-only atmosphere and treats absorption afterward by convolving the individual photon paths with the associated absorption profile.

Using a simple 1-D model, we demonstrated that these two different approaches give results that are identical in a statistical sense for a wide range of scenarios.

A more in depth comparison between the two approaches is made using the spherical implementation of McSCIA. Several scenario studies show that the ET and the SSA approaches give equivalent results, even for extreme cases.

To our knowledge this is the first implementation of the Equivalence Theorem in a 3-D spherical RT model. This approach allows us to study the radiance field, simulated for a particular scattering geometry, as a function of 3-D atmospheric absorption features. For simulations with $10^{6}$ photons, the relative error of McSCIA is normally well under $0.1 \%$. However, due to the required storage of all the individual photon paths, the computational burden is still too high for operational applications. However, McSCIA already acts as a reference model for faster approximate radiative transfer codes (e.g. Walter et al., 2006). Moreover, McSCIA is one of the few models that allow the study of 3-D varying absorption features in a spherical atmosphere. Currently, McSCIA is used to simulate 3-D absorption features for nadir and limb satellite measurements.

\section{Appendix A}

\section{McSCIA Ray tracing algorithm description}

This Appendix gives the formulas needed to create a MC RT ray tracing model, without absorption, that is treated in Sect. 4.1.

\section{A1 Radiative transfer laws and random numbers}

Since RT processes are statistical in nature, most quantities in transfer theory can be easily interpreted as probabilities, or probability distributions.

In the Appendix the wavelength dependence has been omitted from the formulas to improve their readability.

The core is the fundamental principle of Monte Carlo simulations (Cashwell and Everett, 1959; Marshak and Davis, 
2005). For the continuous case, let $p(x)$ be the normalised probability (PF), with $a \leq x<b$ :

$\int_{a}^{b} \mathrm{~d} \xi p(\xi)=1$.

Then $p(x) \mathrm{d} x$ is the probability of $x$ lying between $x$ and $x+\mathrm{d} x$. The cumulative probability function $P(x)(\mathrm{CPF}) \mathrm{de}-$ termines $x$ uniquely as a function of the random number $\mathcal{R}$ :

$\mathcal{R}=P(x)=\int_{a}^{x} \mathrm{~d} \xi p(\xi)$.

Moreover, if $\mathcal{R}$ is uniformly distributed on $0 \leq \mathcal{R}<1$, then $x$ falls with frequency $p(x) \mathrm{d} x$ in the interval $(x, x+\mathrm{d} x)$.

Thus, the CPF is the quantity that relates a random number to physical processes. We will show in the next sections some examples of this relation, guided by the processes that are implemented in McSCIA.

\section{A2 Photon path length}

The normalised probability $\operatorname{PF}(s)$ that a photon will travel through a medium from point 0 to point $s$ (see e.g. Oikarinen et al., 1999; Marshak and Davis, 2005; Cahalan and Davies, 2000), following Lambert-Beer's law (Liou, 1980), is

$\operatorname{PF}(s)=\frac{\exp \left(-\tau_{s}\right)}{\int_{0}^{\infty} \mathrm{d} \tau_{s}^{\prime} \exp \left(-\tau_{s}^{\prime}\right)} \quad(0 \leq \operatorname{PF}(s) \leq 1)$.

The fundamental principle of Monte Carlo simulations must be applied to statistically derive the optical depth travelled, so $\mathcal{R}_{\tau}$ must be equal to the normalised CPF

$\mathcal{R}_{\tau}=\frac{\int_{0}^{\Delta \tau} \mathrm{d} \tau_{s}^{\prime} \exp \left(-\tau_{s}^{\prime}\right)}{\int_{0}^{\infty} \mathrm{d} \tau_{s}^{\prime} \exp \left(-\tau_{s}^{\prime}\right)}=1-\exp (-\Delta \tau)$

$0 \leq \mathcal{R}_{\tau}<1$.

The statistical optical depth travelled before the next collision is then given by

$\Delta \tau=-\ln \left(1-\mathcal{R}_{\tau}\right) \quad 0 \leq \mathcal{R}_{\tau}<1$.

But, since $1-\mathcal{R}_{\tau}$ is still a random number between 0 and 1 , Eq. (A4) can be rewritten as

$\Delta \tau=-\ln \left(\mathcal{R}_{\tau}^{\prime}\right) \quad 0<\mathcal{R}_{\tau}^{\prime} \leq 1$

With a backward MC in limb view it is advantageous to bias this distribution, permitting the photons only to scatter in $\left(0, \Delta \tau_{\max }\right]$, so that the biased sampled photons do not leave the atmosphere at the first scattering event. In that case the sampling would be

$\Delta \tau=-\ln \left[1-\mathcal{R}_{\tau}\left(1-\exp \left(-\Delta \tau_{\max }\right)\right)\right]$

$0 \leq \mathcal{R}_{\tau}<1$.

To account for this bias the weight of the photon has to be multiplied by $1-\exp \left(-\Delta \tau_{\max }\right)$, which simply states that a fraction of $\left(1-\exp \left(-\Delta \tau_{\max }\right)\right)$ of all the photons leave the atmosphere after being emitted from the satellite.

\section{A3 Scattering angles}

When a photon is scattered by molecules (Rayleigh scattering) or aerosols and droplets (Mie scattering) or is reflected from the ground, its direction changes. In order to find the new direction the scattering azimuth and zenith angles have to be simulated (see e.g. Oikarinen et al., 1999; Marshak and Davis, 2005; Cahalan and Davies, 2000), in a statistical sense.

The rotation of the scattering angles $\Theta$ and $\Phi$ with respect to the atmospheric coordinate system are discussed in Sect. A3.5.

\section{A3.1 Scattering azimuth angle}

The scattering azimuth angle, which determines the plane of the scattering event relative to the reference direction, is uniformly distributed, that is

$p_{\Phi}(\Phi)=\frac{1}{2 \pi}$

so that applying Eq. (A1)

$\Phi=2 \pi \mathcal{R}_{\Phi} \quad 0 \leq \mathcal{R}_{\Phi}<1$.

\section{A3.2 Scattering zenith angle}

The scattering zenith angle $\Theta$ (relative to the incident direction) is determined from the scattering phase function. In the rest of the Appendix we will use, for simplicity, this notation

$\mu=\cos (\Theta)$.

The rotation of the scattering angles angles $\Theta$ and $\Phi$ with respect to the atmospheric coordinate system are discussed in Sect. A3.5

\section{Rayleigh scattering}

For Rayleigh scattering by air, the phase function for unpolarised light is (Liou, 1980):

$P(\mu)=\frac{3}{4}\left(1+\mu^{2}\right) \quad \mu \in[-1,1]$.

In this case Eq. (A1) becomes

$\mathcal{R}_{\mu}=\frac{1}{2}+\frac{1}{8}\left(3 \mu+\mu^{3}\right) \quad 0 \leq \mathcal{R}_{\mu}<1$.

This is a third order equation that can be solved exactly. Since the quadratic term is absent it is possible to use the "Formula Cardanica". Equation (A10) can be rewritten as:

$\mu^{3}+p \mu+q=0$

$p=3$ and $q=4-8 \mathcal{R}_{\mu} \quad 0 \leq \mathcal{R}_{\mu}<1$

Since

$\Delta=\frac{q^{2}}{4}+\frac{p^{3}}{27}>0 \quad 0 \leq \mathcal{R}_{\mu}<1$ 
there is a real solution and two complex solutions. The real solution is:

$\mu=(\sqrt[3]{a-q}-\sqrt[3]{a+q}) \sqrt[3]{\frac{1}{2}}$

$q=4-8 \mathcal{R}_{\mu}$ and $a=\sqrt{q^{2}+4}$

\section{Henyey-Greenstein scattering}

For scattering of photons in the UV-vis region on aerosols and droplets, Mie scattering theory (see e.g. Lenoble, 1993) has to be used. A Mie scattering phase function is generally complicated, but a reasonable approximation is the HenyeyGreenstein function

$P(\mu)=\frac{1-g^{2}}{\left(1+g^{2}-2 g \mu\right)^{\frac{3}{2}}}$

where $g$ is the asymmetry factor. The relation between the scattering angle and the random number obtained using the fundamental principle of Monte Carlo simulations is

$\mu=\frac{1}{2 g}\left[1+g^{2}-\left(\frac{1-g^{2}}{1-g+2 g \mathcal{R}_{\mu}}\right)^{2}\right]$

$0 \leq \mathcal{R}_{\mu}<1$

where $g$ is the asymmetry factor of the phase function defined as

$g=<\mu>=\frac{\int_{-1}^{+1} \mu p(\mu) \mathrm{d} \mu}{\int_{-1}^{+1} p(\mu) \mathrm{d} \mu}$.

\section{Mixed phase function}

When the model has to take into account scattering from more than one type of particles, a mixed phase function has to be used (Oikarinen et al., 1999). Suppose $k^{\text {ext }}(\boldsymbol{x})$ represents the total volume extinction coefficient: absorption and scattering both by molecules and particles. In general, the profile will be a function of the 3-D position $\boldsymbol{x}$.

McSCIA needs to separate the extinction coefficient in scattering and absorption coefficients:

$k^{\mathrm{ext}}(\boldsymbol{x})=k^{\mathrm{abs}}(\boldsymbol{x})+k^{\mathrm{sca}}(\boldsymbol{x})$

and then to separate the scattering coefficients in molecular and aerosol scattering

$k^{\mathrm{sca}}(\boldsymbol{x})=k^{\mathrm{sca} \_\mathrm{mol}}(\boldsymbol{x})+k^{\mathrm{sca} \_ \text {aer }}(\boldsymbol{x})$.

The single scattering albedo is defined as usual

$\omega(\boldsymbol{x})=\frac{k^{\mathrm{sca}}(\boldsymbol{x})}{k^{\mathrm{ext}}(\boldsymbol{x})}$.
The ratio between molecular and total scattering is then represented by

$f^{\mathrm{sca}}(\boldsymbol{x})=\frac{k^{\mathrm{sca} \_\mathrm{mol}}(\boldsymbol{x})}{k^{\mathrm{sca}}(\boldsymbol{x})}$.

For molecular scattering Eq. (A9) is employed, and for aerosol scattering Eq. (A13). At a scattering event first a random number is drawn to decide whether the scattering will be molecular or from aerosol:

$\mathcal{R}_{\text {sca }} \leq f^{\text {sca }} \Rightarrow$ use Eq. (A12)

$\mathcal{R}_{\text {sca }}>f^{\text {sca }} \Rightarrow$ use Eq. (A14)

When the exact scattering probability of scattering towards the sun has to be computed (see Eq. A26) a mixed phase function expression is used

$$
\begin{aligned}
P(\boldsymbol{x}, \mu) & =P^{\mathrm{mol}}(\mu) \cdot f^{\mathrm{sca}}(\boldsymbol{x})+ \\
& +P^{\mathrm{aer}}(\mu) \cdot\left(1-f^{\mathrm{sca}}(\boldsymbol{x})\right)
\end{aligned}
$$

While the phase function for molecular scattering $P^{\mathrm{mol}}$ and aerosol scattering $P^{\text {aer }}$ are taken to be independent of the position $\boldsymbol{x}$, the mixed phase function is a function of the position $\boldsymbol{x}$.

\section{A3.3 Lambertian surface reflection}

When a photon reaches the surface and is reflected, the new direction is uniformly sampled. Thus Eq. (A1) becomes

$\mathcal{R}_{\mu}=\int_{0}^{\mu} \mathrm{d} \mu^{\prime} \mu^{\prime} \quad 0 \leq \mathcal{R}_{\mu} \leq 1$

The relation between the scattering angle and the random number is then given by

$\mu=\sqrt{\mathcal{R}_{\mu}} \quad 0 \leq \mathcal{R}_{\mu} \leq 1$.

As for atmospheric scattering, the azimuth angle for surface reflection is calculated using Eq. (A8). Since the direction of reflection it is defined only by the random number, it is easy to introduce a 2-D variability. This is only accounted for in the value of albedo $a\left(\boldsymbol{x}_{j}\right)$ that is used to calculate $w_{i}^{\mathrm{alb}}$ (see Eq. 4).

\section{A3.4 Scattering angle to the sun}

Equation (A26) requires the angle between the photon direction and the solar rays. This angle can be calculated in spherical geometry as

$$
\begin{array}{r}
\mu_{s_{i}}\left(\theta_{i}^{\mathrm{dir}}, \phi_{i}^{\mathrm{dir}}, \theta_{i}^{\text {sun }}, \phi_{i}^{\text {sun }}\right)=\cos \left(\theta_{i}^{\mathrm{dir}}\right) \cos \left(\theta_{i}^{\text {sun }}\right)+ \\
+\sin \left(\theta_{i}^{\text {dir }}\right) \sin \left(\theta_{i}^{\text {sun }}\right) \cos \left(\phi_{i}^{\text {dir }}-\phi_{i}^{\text {sun }}\right) .
\end{array}
$$

The directional angles $\theta_{i}^{\text {dir }}$ and $\phi_{i}^{\text {dir }}$ are different at each scattering event, while the solar angles $\theta^{\text {sun }}$ and $\phi^{\text {sun }}$ are always the same, since a global reference system is used.

Alternatively, given positions $\boldsymbol{x}_{i-1}, \boldsymbol{x}_{i}$ and $\boldsymbol{x}_{i}^{\text {out }}$ (see Fig. A1) the vector formula can be used

$\mu_{s_{i}}\left(\theta_{i}^{\mathrm{dir}}, \phi_{i}^{\mathrm{dir}}, \theta_{i}^{\mathrm{sun}}, \phi_{i}^{\mathrm{sun}}\right)=\overrightarrow{\boldsymbol{x}_{i-1} \boldsymbol{x}_{i}} \cdot \overrightarrow{\boldsymbol{x}_{i} \boldsymbol{x}_{i}^{\mathrm{out}}}$. 


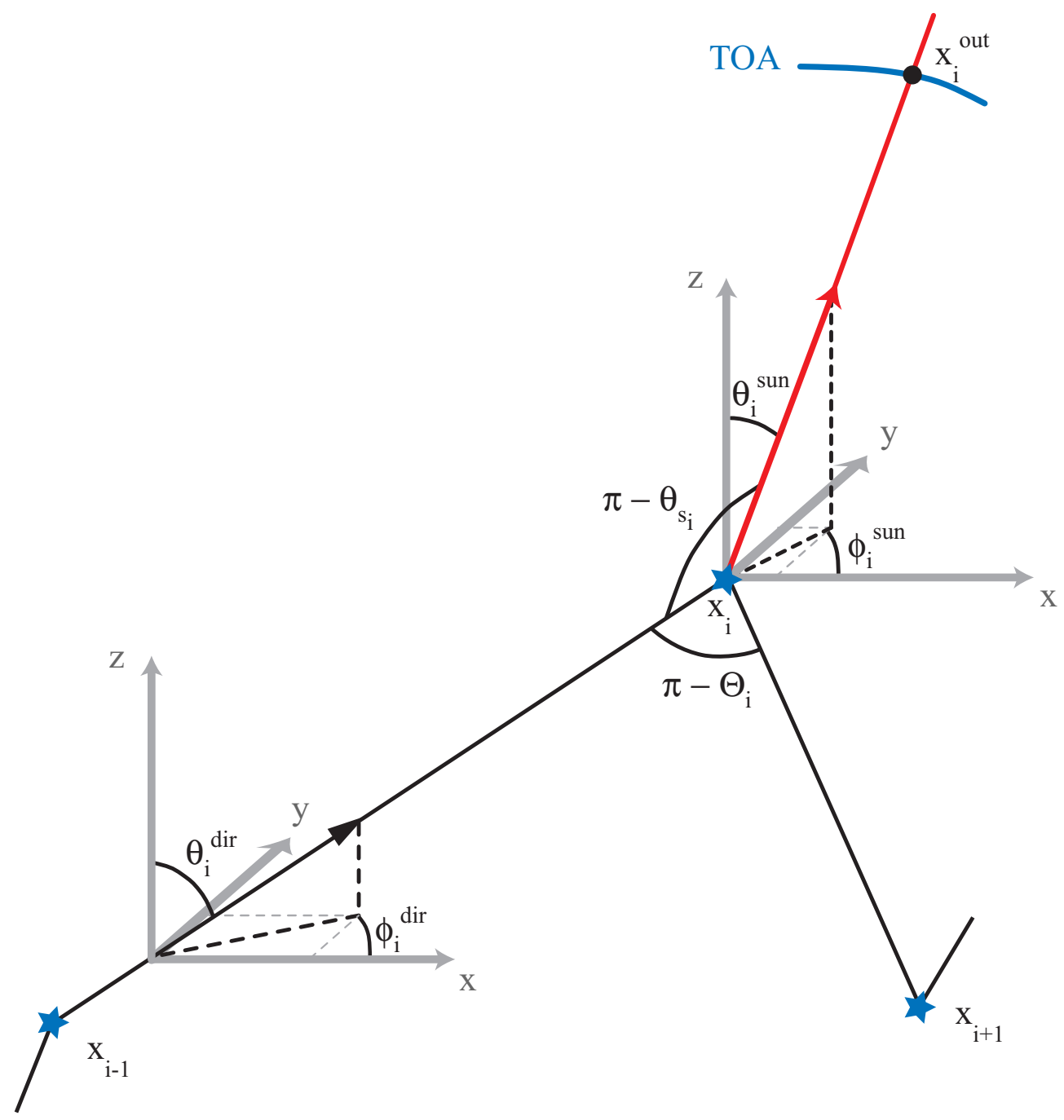

Fig. A1. Zoom of Fig. 1, in particular of points $x_{3}$ and $x_{4}$. For clarity of drawing not all scales are identical. The scattering probability towards the sun following the direction $x_{i}-x_{i}^{\text {out }}$ is calculated evaluating the phase function in $\mu_{s_{i}}$ (Eq. A26). Computation of angle $\theta_{s_{i}}$ requires the directional and solar angles $\theta_{i}^{\mathrm{dir}}, \phi_{i}^{\mathrm{dir}}, \theta_{i}^{\text {sun }}, \phi_{i}^{\text {sun }}$ (see Eq. A23). The photon travels from position $x_{i-1}$ to $x_{i}$ and the angles are defined in the global reference system $\mathrm{x}, \mathrm{y}, \mathrm{z}$.

\section{A3.5 New photon direction}

Calculation of the new direction of a photon after a scattering event, requires the old direction and scattering angles. The latter are calculated using formulas (A8) and (A12), (A14) or (A22). In the local reference system of the old direction the new direction is calculated by two successive rotations of $\Theta$ and $\Phi$, respectively, as illustrated in Fig. A2. This new direction is then rotated back from the local reference system to the global reference system.

\section{A4 Ray tracing module}

Since the field of view (FOV) of the satellite is normally narrow usually a backward MC approach is preferred (Collins et al., 1972; Adams and Kattawar, 1978; Marchuk et al., 1980; Lenoble, 1985; Oikarinen et al., 1999). The principle behind this method is illustrated in Fig. 1. With a time reversal, photons emerge from the detector and are traced through the atmosphere. The fate of these photons is influenced by scattering on air molecules (Rayleigh), aerosols (Mie), absorption by trace gases, and surface reflection. Since in the UV-vis wavelength region emission can be generally ignored, the only source of light is the sun. However, the chance that a photon leaves the atmosphere exactly in the direction of the sun are extremely low. Thus, many photons would be needed to obtain a statistically meaningful result. Luckily a much faster convergence can be obtained by using the local estimate technique (Marchuk et al., 1980; Davis 
et al., 1985; Marshak and Davis, 2005). It consists in calculating the contribution of every photon at each scattering event (see Fig. 1). If we follow the $j$-th photon (e.g. the one of Fig. 1), at each scattering position $\boldsymbol{x}_{i}$, the probabilities that the photons escape in the direction of the sun are calculated. For a scattering-only atmosphere the radiance contribution of this photon at the $\mathrm{i}$-th scattering event is given by

$I_{i}=S_{i} \cdot T_{i}$.

$S_{i}$ is the scattering probability towards the sun

$S_{i}=P\left(\mu_{S_{i}}\right) / 4 \pi$,

where $\mu_{s_{i}}$ is the cosine of the scattering angle towards the sun, $\theta_{s_{i}}$ (see also Fig. 1, Eqs. A23 and A24). $P(\mu)$ is the scattering phase function, normalised over the solid angle:

$\int_{4 \pi} \frac{P(\mu)}{4 \pi} \mathrm{d} \Omega=1$

where $\mathrm{d} \Omega$ is the infinitesimal element of a solid angle, $\mathrm{d} \Omega=\mathrm{d} \mu \mathrm{d} \phi$. The transmittance $T_{i}$ in Eq. (A25) from the photon position to the TOA in the direction of the sun, which takes into account the intensity scattered out of the ray, is given by:

$T_{i}=e^{-\tau_{\mathrm{sca}}}$

where $\tau_{\mathrm{sca}_{\mathrm{i}}}$ is the outgoing optical thickness:

$\tau_{\mathrm{sca}_{\mathrm{i}}}=\int_{s} k_{\mathrm{sca}}(\boldsymbol{s}) \mathrm{d} \boldsymbol{s}$.

where $\boldsymbol{s}$ is the line connecting the scattering position $\boldsymbol{x}_{i}$ to $\boldsymbol{x}_{i}^{\text {out }}$, the position where the photon leaves the atmosphere towards the sun. $k_{\text {sca }}$ is the scattering coefficient.

The result of this ray-tracing procedure is a number of scattering positions $\left(\boldsymbol{x}_{i}, i(=) 1,5\right.$ in Fig. 1$)$ of all the photons that travel through the model atmosphere. To find the new positions and directions of the photons after each scattering event, we use the formulae described in the Appendix, following the algorithm illustrated by the flow chart of Fig. 2a. For the first scattering event in limb geometry we use the biased Eq. (A6) instead of (A5). In this way all photons remain in the atmosphere after the first scattering and no photon is lost directly to space. As described by (Marchuk et al., 1980), not using this biasing would result in very bad statistics for the limb case.

The photons can only end their trajectories if they are scattered into space.

The normalised radiance $I$ measured by the satellite is given by the sum of all contributions at the scattering events $i$ of photon $j$, divided by the total number of photons simulated $\left(N_{\text {tot }}\right)$ multiplied by $\pi$

$I=\frac{\pi}{N_{\mathrm{tot}}} \sum_{j=1}^{N_{\mathrm{tot}}} \sum_{i=1}^{N_{\mathrm{sca}}(j)} I_{i, j}$.

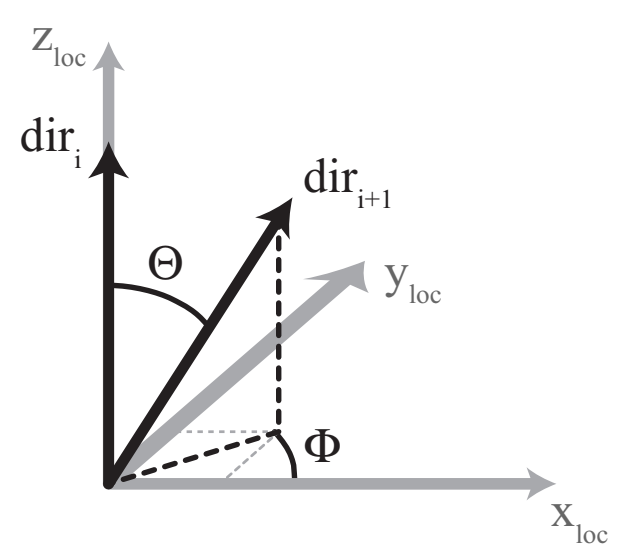

Fig. A2. Scattering angles. The new direction $\operatorname{dir}_{i+1}$ of the photon is calculated by rotating the old direction $\operatorname{dir}_{i}$ by two angles $\Theta$ and $\Phi$ in the local system $\left(\mathrm{x}_{\text {loc }}, \mathrm{y}_{\text {loc }}, \mathrm{z}_{\text {loc }}\right)$.

The number of scattering events that a photon undergoes, $N_{\text {sca }}(j)$, can be different for each photon. The factor $\pi$ in equation above is needed if we assume that the monochromatic incident solar flux through a surface unit perpendicular to the incident solar beam is $\pi \mathrm{W} \mathrm{m}^{-2}$. Thus the normalised radiance is expressed in $\mathrm{sr}^{-1}$. To obtain the actual value of the radiance, one must multiply it by the extraterrestrial solar spectral irradiance.

It is useful to calculate also the single scattering radiance, $I_{S S}$. In this case only the contribution given by the first scattering event of a photon is considered. $I_{S S}$ is given by

$I_{S S}=\frac{\pi}{N_{\text {tot }}} \sum_{j=1}^{N_{\text {tot }}} I_{1, j}$.

In addition to $I_{S S}$ we will refer to the radiance given by total scattering (TS) simply as radiance $I$ or $I_{T S}$. We choose this terminology, because for the widely used term multiple scattering (MS) it is not always clear if it refers to the total scattering (MS=TS) or only to the part that is scattered more than once (MS=TS-SS). The SS component, when calculated for nadir with ground albedo greater than zero, will contain the radiation scattered only once by the ground or the atmosphere.

\section{Appendix B}

\section{Simple 1-D MCRT model}

To demonstrate in a didactic way that the ET and SSA approach have equivalent results, we have developed a simple 1-D MCRT model.

The atmosphere is plane-parallel (PP), and stretches from the ground to $100 \mathrm{~km}$ in height. The phase function that we 
Table B1. Parameters for Eq. (B1) used to generate the atmospheric profiles for the scenarios ET/SSA comparison. The total absorption optical thickness varies from 0 to 7.4 and the total scattering optical thickness varies from 0.08 to 8.0 .

\begin{tabular}{cl}
\hline $\begin{array}{c}z_{\text {scale }} \\
z_{\max }\end{array}$ & $8.0 \mathrm{~km}$ \\
\hline$c_{s}$ & $0.01,0 \mathrm{~km}$ \\
$c_{a}$ & $10^{-4}, 5 \times 10^{-4}, 10^{-3}, 5 \times 10^{-3}, 10^{-2}$, \\
& $5 \times 10^{-2}, 7.5 \times 10^{-2}, 10^{-1}, 2 \times 10^{-1}, 3 \times 10^{-1} \mathrm{~km}^{-1}$ \\
$d$ & $0.01 \longrightarrow 1 \mathrm{~km}$ with step $0.01 \mathrm{~km}, 5,10 \mathrm{~km}$ \\
\hline
\end{tabular}

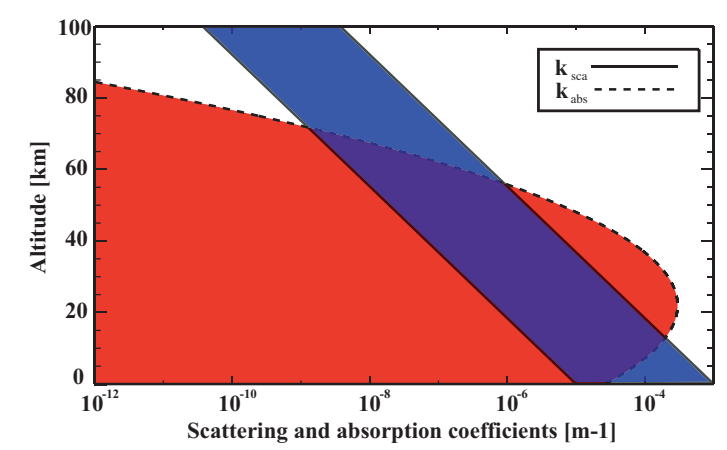

Fig. B1. Scattering coefficient (full line) and absorption coefficient (dashed line) as a function of the altitude used for the demonstration of the ET in 1-D. The coloured region between the two full lines represents the area in which the scattering profiles were varied. The colored region between the dashed line and the axes represents the area in which the absorbing profiles were varied. Optical properties were calculated with Eq. (B1) with parameters from Table B1.

choose is a fully backscattering one, i.e. it inverts the direction of the photon at each scattering event. Therefore, the photons move along a single line (1-D). The optical parameters are described by the absorption and scattering coefficients and single scattering albedo

$$
\begin{aligned}
& k_{\mathrm{sca}}(z)=c_{s} \exp \left(-\frac{z}{z_{\text {scale }}}\right), \\
& k_{\mathrm{abs}}(z)=c_{a} \exp \left(-\frac{\left(z-z_{\max }\right)^{2}}{2 d^{2}}\right), \\
& \omega(z)=\frac{k_{\mathrm{sca}}}{k_{\mathrm{sca}}+k_{\mathrm{abs}}}
\end{aligned}
$$

where $c_{a}\left[\mathrm{~km}^{-1}\right], c_{s}\left[\mathrm{~km}^{-1}\right], d[\mathrm{~km}], z_{\text {scale }}[\mathrm{km}], z_{\max }[\mathrm{km}]$ are parameters specified in Table B1 and $z[\mathrm{~km}]$ is the vertical coordinate. The absorption layer formulated in this way, mimics the absorption of UV in the "ozone" layer.

In this simple model the weight of the photon (Eq. 5 for ET and Eq. 6 for SSA) is reduced to describe absorption. The ground is supposed to have albedo 0 , so the photons end their trajectories either when they hit the ground or as they leave the atmosphere.
In the ET method, the scattering optical depth is used as the vertical coordinate

$\tau_{\mathrm{sca}}(z)=\int_{z}^{z_{\mathrm{TOA}}} k_{S}\left(z^{\prime}\right) \mathrm{d} z^{\prime}$

where $z_{\mathrm{TOA}}$ is the coordinate of the TOA. The photon is initialised at the TOA in a downward direction and its weight is initialized to 1 . The new position is calculated using the Eq. (A4). If the photon is still in the atmosphere its weight (Eq. 5) is calculated. The process starts with an ensemble of photons (e.g. $10^{6}$ ) and iterates until all photons leaves the atmospheres. In the case the photon would end its trajectory, on the ground or in space, the weight of the photon contributes to the measured flux at the boundaries of the domain.

Instead, if the SSA approach is used, the total optical depth is used as vertical coordinate:

$\tau_{\text {ext }}(z)=\int_{z}^{z_{\text {TOA }}}\left(k_{\text {abs }}\left(z^{\prime}\right)+k_{\text {sca }}\left(z^{\prime}\right)\right) \mathrm{d} z^{\prime}$.

It is important to realise that from a numerical point of view a completely different atmosphere is simulated: it is optically thicker in the SSA case than in the ET case. Another difference between the two methods is the way in which the weight is calculated: in the ET case the weight due to absorption is $\exp \left(-\Delta \tau_{\mathrm{abs}}\right)$, where $\Delta \tau_{\mathrm{abs}}$ is the absorption optical thickness between two subsequent scattering events. In the SSA approach, the single scattering albedo evaluated at the scattering position is used to reduce the photon weight.

Acknowledgements. This research was supported by the GO EO041 grant from the Space Research Organisation of the Netherlands (SRON). The authors would like to thank H. Walter and J. Landgraf for many insightful discussions and the inter-comparison work. We are grateful also to R. Loughman for providing us the data necessary for the model validation. We would like to thanks all the reviewers, that with their comments helped us to make a better paper.

Edited by: F. J. Dentener

\section{References}

Adams, C. N. and Kattawar, G. W.: Radiative transfer in spherical shell atmospheres 1. Rayleigh Scattering, Icarus, 35, 139-151, 1978.

Berk, A., Bernstein, L. S., and Robertson, D. C.: MODTRAN: A moderate resolution model for LOWTRAN-7, GL-TR-89-0122, Geophysics Laboratory, Hanscom AFB, MA 01732, 1989.

Bovensmann, H., Burrows, J. P., Buchwitz, M., Frerick, J., Noel, S., Rozanov, V. V., Chance, K. V., and Goede, A. P. H.: SCIAMACHY: Mission Objectives and Measurement Modes, J. Atmos. Sci., 56, 127-150, 1999.

Burrows, J. P., Weber, M., Buchwitz, M., Rozanov, V., LadstatterWeissenmayer, A., Richter, A., de Beek, R., Hoogen, R., Bramstedt, K., Eichmann, K., Eisinger, M., and Perner, D.: The Global Ozone Monitoring Experiment (GOME): Mission concept and first scientific results, J. Atmos Sci., 56, 151-175, 1999. 
Cahalan, R. and Davies, R.: Intercomparison of three-dimensional radiation codes (I3RC): Proceedings of the first and second international workshops., University of Arizona Press, 2000.

Cahalan, R., Ridgway, W., Wiscombe, W., Gollmer, S., and Harshvardhan: Independent pixel and Monte Carlo estimates of stratocumulus albedo, J. Atmos. Sci., 51, 3776-3790, 1994.

Cashwell, E. D. and Everett, C. J.: Monte Carlo Method for random walk problems, Pergamon Press, 1959.

Collins, D. G., Blattner, W. G., Wells, M. B., and Horak, H. G.: Backward Monte Carlo calculations of the polarization characteristics of the radiation emerging from spherical shell atmospheres, Appl. Opt., 11, 2684-2696, 1972.

Davis, J., McKee, T., and Cox, S.: Application of the Monte Carlo method to problems in visibility using a local estimate: an investigation, Appl. Opt., 24, 3193-3205, 1985.

de Haan, J., Bosma, P., and Hovenier, J.: The adding method for multiple scattering calculations of polarized light, Astron. Astrophys., 183, 371-391, 1987.

Feigelson, E. (Ed.): Radiation in a cloudy atmosphere, Kluwer, p. 308, 1984.

Flittner, D. E., Bhartia, P. K., and Herman, B. M.: O3 profiles retrieved from limb scatter measurements: Theory, Geophys. Res. Lett., 27, 2601-2604, 2000.

Heidinger, A. and Stephens, G. L.: Molecular Line Absorption in a Scattering Atmosphere. Part II: Application to remote Sensing in the $\mathrm{O}_{2}$ A band, J. Atmos. Sci., 57, 1615-1634, 2000.

Heidinger, A. and Stephens, G. L.: Molecular Line Absorption in a Scattering Atmosphere. Part III: Pathlength Characteristics and Effects of Spatially Heterogeneous Clouds, J. Atmos. Sci., 59, 1641-1654, 2002.

Irvine, W.: The formation of absorption bands and the distribution of photon optical paths in a scattering atmosphere, Bulletin of the Astronomical Institutes of The Netherlands, 17, 266-279, 1964.

Jacob, D. J.: Introduction to Atmospheric Chemistry, Princeton University Press, 1999.

Kaiser, J.: Atmospheric Parameter Retrieval from UV-vis-NIR Limb Scattering Measurements, Logos Verlag, Berlin, Germany, 2002.

Kaiser, J., von Savigny, C., Eichmann, K.-U., Nol, S., Bovensmann, H., Frerick, J., and Burrows, J.: Satellite Pointing Retrieval from Atmospheric Limb Scattering of Solar UV-B Radiation, Can. J. Phys., 82, 1041-1052, 2004.

Kattawar, G. W. and Adams, C. N.: Radiative transfer in spherical shell atmospheres, II, asymmetric phase function, Icarus, pp. 436-449, 1978.

Lenoble, J.: Radiative transfer in scattering and absorbing atmospheres: Standard computational procedures, A. Deepak Publishing, Hampton, Virginia, 1985.

Lenoble, J.: Atmospheric Radiative Transfer, A. Deepak Publishing, Hampton, Virginia, 1993.

Levelt, P. F., van den Oord, G. H. J., Dobber, M. R., Malkki, A., Visser, H., de Vries, J., Stammes, P., Lundell, J., and Saari, H.: The Ozone Monitoring Instrument, IEEE Trans. Geo. Rem. Sens., Special Issue on the EOS-Aura mission, 44(5), 11991208, 2006.

Liou, K.-N.: An introduction to atmospheric radiation, Academic Press Inc., 1980.

Loughman, R., Griffioen, E., Oikarinen, L., Postylyakov, O., Rozanov, A., Flittner, D., and Rault, D.: Comparison of radia- tive transfer models for limb-viewing scattered sunlight measurements, J. Geophys. Res., D, Atmos., 109(D18), 6303, doi:10.1029/2003JD003854, 2004.

Marchuk, G. I., Mikhailov, G. A., Nazaraliev, M. A., Dearbinjan, R. A., Kargin, B. A., and Elepov, B. S.: The Monte Carlo methods in atmospheric optics, vol. 12 of Springer ser. in Opt. Sci., Springer Verlag, 1980.

Marshak, A. and Davis, A.: 3D radiative transfer in cloudy atmospheres, Springer Berlin, Heidelberg, New York, 2005.

Mount, G. H., Rusch, D. W., Noxon, J. F., Zawodny, J. M., and Barth, C. A.: Measurements of stratospheric NO2 from the SME satellite, J. Geophys. Res., 89, 1327-1340, 1984.

O'Hirok, W. and Gautier, C.: A three-dimensional radiative transfer model to investigate the solar radiation within a cloudy atmosphere. Part I: Spatial effects., J. Atmos. Sci., 55, 2162-2179, 1998.

Oikarinen, L., Sihvola, E., and Kyrola, E.: Multiple scattering radiance in limb-viewing geometry, J. Geophys. Res., 104, 31261 $31274,1999$.

Partain, P. T., Heidinger, A. K., and Stephens, G. L.: High spectral resolution atmospheric radiative transfer: Application of the equivalence theorem, J. Geophys. Res., 105, 2163-2177, 2000.

Postylyakov, O. V.: Linearized vector radiative transfer model $\mathrm{MCC}++$ for a spherical atmosphere, J. Quant. Spectr. Radiat. Trans., 88, 297-317, 2004.

Postylyakov, O. V., Kyrola, E., and Oikarinen, L.: Fine comparison of radiative transfer models for scattering limb simulation, EGS AGU - EUG Joint Assembly, Abstracts from the meeting held in Nice, France, 6-11 April 2003, abstract \#5566, pp. 5566-5567, 2003.

Rozanov, A., Bovensmann, H., Bracher, A., Hrechany, S., Rozanov, V., Sinnhuber, M., Stroh, F., and Burrows, J.: NO2 and BrO vertical profile retrieval from SCIAMACHY limb measurements: Sensitivity studies, Adv. Space Res., 2005.

Rusch, D. W., Mount, G. H., Barth, C. A., Thomas, R. J., and Callan, M. T.: Solar mesosphere explorer ultraviolet spectrometer: measurements of ozone in the $1.0-0.1 \mathrm{mb}$ region, J. Geophys. Res., 89, 11 677-11678, 1984.

Segers, A. J., von Savigny, C., Brinksma, E. J., and Piters, A. J. M.: Validation of IFE-1.6 SCIAMACHY limb ozone profiles, Atmos. Chem. Phys., 5, 3045-3052, 2005, http://www.atmos-chem-phys.net/5/3045/2005/.

Sioris, C., Kurosu, T., Martin, R., and Chance, K.: Stratospheric and tropospheric NO2 observed by SCIAMACHY: first results, Adv. Space Res., 34, 780-785, 2004.

Spada, F. and Krol, M. C.: McSCIA: a Monte Carlo model for analysis of 3D features in a spherical atmosphere, in IRS 2004: Current Problems in Atmospheric Radiation, A. Deepak Publishing, Hampton, Virginia, 2005.

Spanier, J. and Gelbard, E. M.: Monte Carlo principles and Neutron Transport Problems, Addison Wesley, 1969.

Stammes, P.: Spectral radiance modelling in the UV-Visible range, in IRS 2000: Current problems in Atmospheric Radiation, pp. 385-388, A. Deepak Publ., Hampton, VA, 2001.

Stammes, P., Levelt, P., de Vries, J., Visser, H., Kruizinga, B., Smorenburg, C., Leppelmeier, G., and Hilsenrath, E.: Scientific requirements and optical design of the Ozone Monitoring Instrument on EOS-CHEM, in: Proceedings of SPIE Conference on Earth Observing Systems IV, vol. 3750, p. 221-232, SPIE, 1999. 
Stephens, G. L. and Heidinger, A.: Molecular Line Absorption in a Scattering Atmosphere. Part I: Theory, J. Atmos. Sci., 57, 15991614, 2000.

van de Hulst, H. C.: Multiple light scattering, Academic press, 1980.

von Savigny, C., Haley, C. S., Sioris, C. E., McDade, I. C., Llewellyn, E. J., Degenstein, D., Evans, W. F. J., Gattinger, R. L., Griffioen, E., Kyrölä, E., Lloyd, N. D., McConnell, J. C., McLinden, C. A., Mégie, G., Murtagh, D. P., Solheim, B., and Strong, K.: Stratospheric ozone profiles retrieved from limb scattered sunlight radiance spectra measured by the OSIRIS instrument on the Odin satellite, Geophys. Res. Lett., 30, 1755-1758, 2003.
Walter, H. and Landgraf, J.: Towards Linearization of Atmospheric Radiative Transfer in Spherical Geometry, J. Quant. Spectr. Radiat. Trans., 95, 175-200, 2005.

Walter, H. H., Landgraf, J., Spada, F., and Doicu, A.: Linearization of a radiative transfer model in spherical geometry, J. Geophys. Res., in press, 2006. 